\title{
A Dual-origami Design Enables the Quasi-sequential Deployment and Bending Motion of Soft Robots and Grippers
}

\author{
Woongbae $\mathrm{Kim}^{1}$, Jaemin Eom ${ }^{1}$, and Kyujin $\mathrm{Cho}^{1}$ \\ ${ }^{1}$ Affiliation not available
}

November 16, 2021

\begin{abstract}
Soft fluidic actuators produce continuous and life-like motions that are intrinsically safe, but current designs are not yet mature enough to enable large deployment with high force and low-cost fabrication methods. Here, soft fluidic actuators with two superimposed origami architectures are reported. Driven by a fluid input, the presented dual-origami soft actuators produce quasi-sequential deployment and bending motion that is guided by unsymmetric unfolding of low-stretchable origami components. The dominance between the deployment and bending can be shifted by varying the unfolding behavior, enabling pre-programming of the motion. The proposed origami-inspired soft actuators are directly fabricated by low-cost fused deposition modeling 3D-printing, and subjected to a heat treatment post-processing to enhance the fluid sealing performance. Finally, soft gripper applications are presented and they successfully demonstrate gripping tasks that each requires strength, delicacy, precision and dexterity. The dual-origami approach offers a design guidance for soft robots to embody grow-and-retract motion with a small initial form factor, promising for applications in next-generation soft robotic systems.
\end{abstract}

Corresponding author(s) Email: kjcho@snu.ac.kr

\section{ToC Figure}



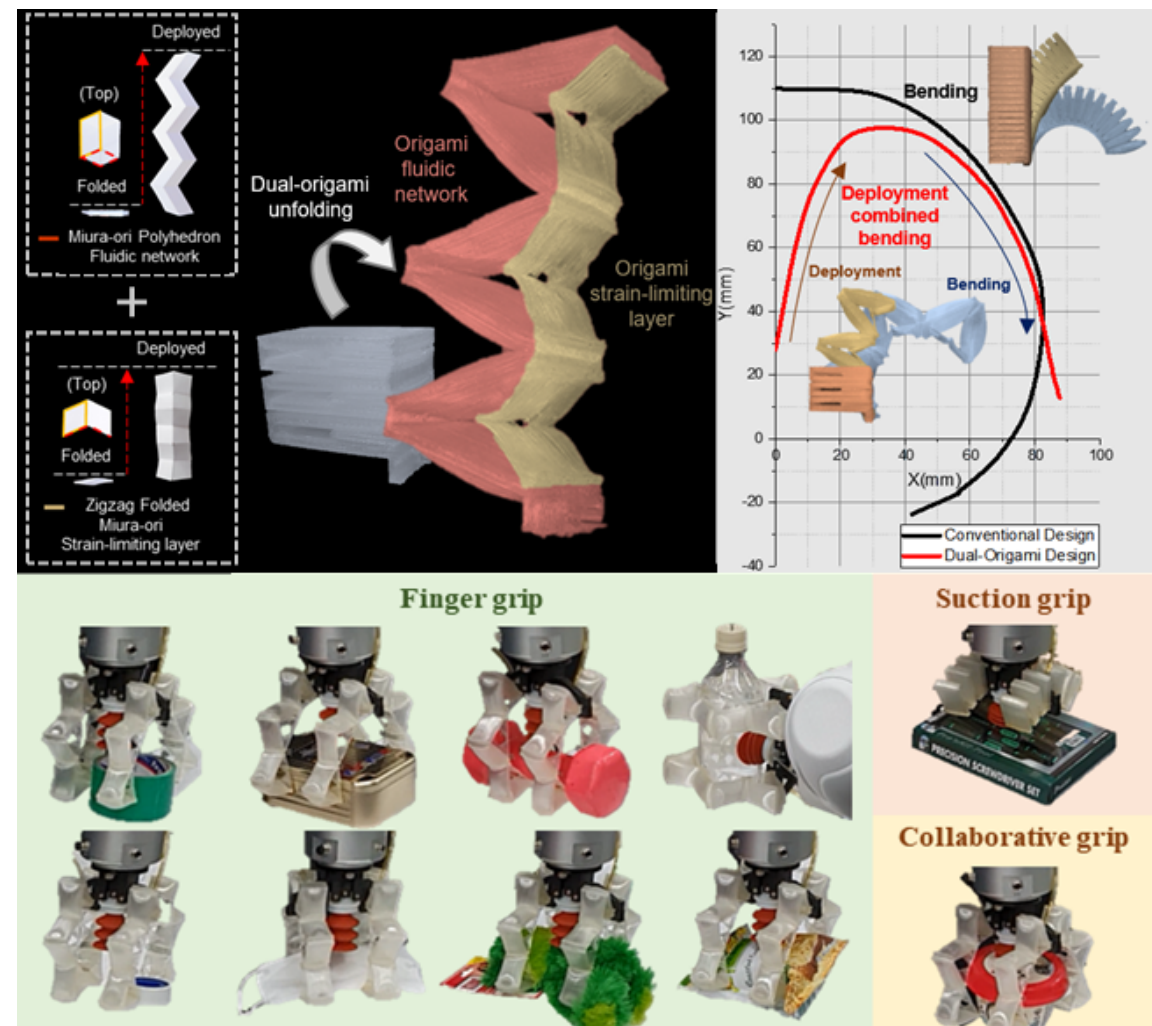

Finger grip
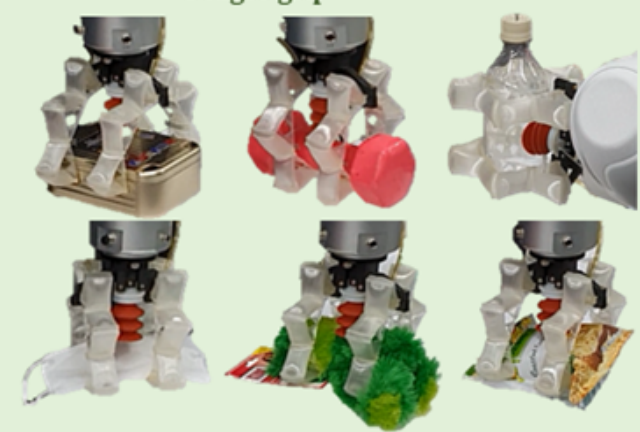

Suction grip

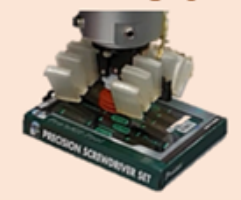

Collaborative grip

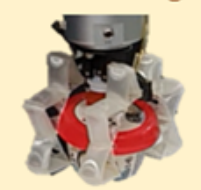

Figure 1: ToC Figure. This work establishes a soft fluidic bending actuator design method where two folded origami architectures are superimposed, to produce pre-programmable quasi-sequential deployment and bending motion in response to single fluid input. Versatile soft gripper utilizing the advantages of compactness and deployment is demonstrated. Low-cost 3D printing and heat treatment post-processing are applied for fabrication of the dual-origami soft actuators.

\section{Introduction}

Advances in soft robotics have presented the way robots interact with human or unstructured environments. In response to external stimuli, such as fluidic pressure, (Marchese et al., 2015; Usevitch et al., 2020; Shah et al., 2021; Katzschmann et al., 2018; Heng et al., 2021; Cianchetti et al., 2014; Hawkes et al., 2017) el signals, (Acome et al., 2018; Xing et al., 2020; Li et al., 2017b) magnetic fields, (Goudu et al., 2020; Joyee and Pan, 2019) and motor-tendon actuation, ${ }^{(M a n t i ~ e t ~ a l ., ~ 2015 ; ~ V i k a s ~ e t ~ a l ., ~ 2016 ; ~ I n ~ e t ~ a l ., ~ 2015) ~ t h e ~ a r c h i t e c t e d ~ s o f t ~ m a t-~}$ ters, mostly elastomers, produce continuous and adaptive motions that allow delicate handling of fragile objects or shape adjusting to unstructured environments. By utilizing the inherent features of softness, soft robots are gradually being used for safety demanding applications or near human applications such as soft grippers, (Goudu et al., 2020; Joyee and Pan, 2019; Brown et al., 2010) soft manipulators, (Xing et al., 2020; Hawkes et al., 2017) mobile robots, (Hawkes et al., 2017; Shah et al., 2021; Katzschmann et al., 2018; Li et al., 2017 assistive wearable robots, ${ }^{(H e n g}$ et al., 2021; In et al., 2015) and minimally invasive surgical tools. (Cianchetti et al., 2014; Runciman et al., 2019) The established design method for soft fluidic robots is to embody anisotropic deformation in soft bodies by carving geometric patterns of extensible fluidic networks and embedding inextensible strain-limiting appendages (e.g., paper strips, fabrics, tendons). (Polygerinos et al., 2017; Ilievski et al., 2011) Consequently, the applied fluid causes asymmetric extension of fluidic networks and strain-limiting appendages, and the whole soft body produces pre-programmed motion such as bending, (Ilievski et al., 2011; Mosadegh et al., 2014; Tang et al., 2020; Yap et al., 2016) twisting, (Connolly et al., 2015) 
and contraction. (Yang et al., 2017; Koizumi et al., 2018) Researches to date have mainly focused on architecting fluidic networks and have achieved rapid actuation, (Mosadegh et al., 2014) high aspect ratio design, (Becker et al., 2020) and high force generation. (Tang et al., 2020; Yap et al., 2016) However, the current design method compels soft robots to be shaped as a long elastomeric beam or a cylinder because their form factors are subordinated to inextensible strain-limiting appendages, which may limit their spatial efficiency, and make it inconvenient for soft fluidic robots to be used with other mechanical elements due to physical interferences.

Origami deployable architectures have unique feature that are folded into compact forms when not in use, and be transformed into large shapes to be functional. This deployment combined functionality provides portability and mobility at the folded state while it can generate large motions at the deployed state. For example, in nature, flying insects such as ladybugs and earwigs fold and retract their hind wings for reduced inertia, and only deploy and flap them during flight. (Deiters et al., 2016; Saito et al., 2017) With additional benefits such as desired shapes or motions which can be robustly guided by kinematics, and a large variety of constituting materials (from compliant paper to steel plate), the origami design has been considered as a promising method for deployable machines with possible applications in mobile robots, (Baek et al., 2020; Lee et al., 2021; Lin et al., 2020) manipulators, (Kim et al., 2018; Suzuki and Wood, 2020) space missions, (Schenk et al., 2014; Pehrson et al., 2020) and biomedical tools. (Suzuki and Wood, 2020; Johnson et al., 2017) Likewise, several researches of soft fluidic robots have adopted origami deployable architectures. Martinez et al developed paper-elastomer composite soft fluidic robots including Yoshimura origami cylinder structure (so-called bellows-like pattern) that grow or bend due to glued facets or an attached strip. ${ }^{\text {(Martinez et al., } 2012)}$ $\mathrm{Li}$ et al presented soft fluid robots in which an origami skeleton is sealed by flexible skin, and negative

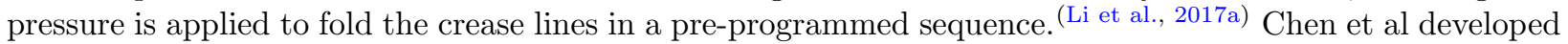
a hybrid actuator with a motor-driven tendon and a fluid-driven asymmetric Yoshimura origami cylinder, in which the effective length of the bending configuration can be changed. (Chen et al., 2021) These existing works successfully imported origami deployable architectures' large range of motion into fluidic networks, while they have not yet focused on designing or programming additional motions beyond kinematics of origami deployment.

Recently, implementation of soft and compliant materials widened the accessible regime of origami folding over origami kinematics. (Faber et al., 2018; Mintchev et al., 2018; Kim et al., 2019) Our previous work, 'a dualmorphing stretchable origami', presented an entirely stretchable origami that deploys by unfolding and then produce additional motion by anisotropic stretching. (Kim et al., 2019) Although this dual-morphing principle that utilizes both morphing principles of unfolding and stretching could achieve extreme shape morphing, there exist several limitations originating from the essential use of highly stretchable materials: i) low force due to incapability of holding high pressure, ii) poor reliability due to non-linearity of materials and Mullins effect, iii) slow response time due to heavy reliance on material response speed iv) difficulty in control due to difficulties in kinematics analysis of stretching behavior, and v) narrow material selection with only a few specific materials (expensive silicone elastomers or high-tech $3 \mathrm{~d}$ printing materials) available. In addition, a complex fabrication process using sacrificial molds, as well as the need for deposition of multiple materials to achieve functional motion (e.g. bending) over deployment and balloon-like bloating, significantly increase fabrication cost. These drawbacks presented challenges for practical usage of dual-morphing principle in real-world applications, highlighting a need for a new design method of a different working principle that does not rely on stretching, broadens the range of applicable materials, and enables large deployment, high force, and low-cost/easy fabrication (the detailed comparison of the dual-morphing principle and the current work is shown in Table 1).

In this paper, we present a soft robot with a small initial form factor that undergoes quasi-sequential deployment and bending motion driven by a single fluid input, enabled by a dual-origami design in which both the fluidic network and the strain-limiting layer are compliant origami architectures. We selectively placed the folded strain-limiting layer in between the facets of the folded fluidic network, building the latter to be more extensible than the former. Accordingly, in an early stage of the response to fluid pressure, the two elements both unfold simultaneously, and the whole body mainly deploys while slight bending also occurs simultaneously, depending on the stiffness of the origami strain-limiting layer. And when the 


\begin{tabular}{|c|c|}
\hline & Dual-origami design \\
\hline Deformation & Extremely large \\
\hline $\begin{array}{l}\text { Morphing } \\
\text { modes }\end{array}$ & 1 (unfolding) \\
\hline $\begin{array}{l}\text { Working } \\
\text { principle }\end{array}$ & Asymmetric unfolding of \\
\hline Motion & $\begin{array}{c}\text { two origami structures } \\
\text { Quasi-sequential } \\
\text { deployment and bending }\end{array}$ \\
\hline $\begin{array}{l}\text { Material } \\
\text { property }\end{array}$ & Flexible but non-stretchable \\
\hline $\begin{array}{l}\text { Material } \\
\text { stiffness }\end{array}$ & $\mathrm{E}_{i} 10 \mathrm{MPa}$ \\
\hline $\begin{array}{l}\text { Number of } \\
\text { materials }\end{array}$ & 1 \\
\hline $\begin{array}{l}\text { Pre- } \\
\text { programming } \\
\text { method }\end{array}$ & $\begin{array}{l}\text { Tuning the relative length and } \\
\text { stiffness of origami structures }\end{array}$ \\
\hline $\begin{array}{c}\text { Applied } \\
\text { pressure level }\end{array}$ & High $(i, 100 \mathrm{kPa})$ \\
\hline Output force & ¿. $3 \mathrm{kgf}$ \\
\hline Reliability & Good \\
\hline Response time & Fast \\
\hline $\begin{array}{l}\text { Kinematically } \\
\text { defined }\end{array}$ & Yes \\
\hline Fabrication & Low-cost 3D printing (FDM) \\
\hline
\end{tabular}
Dual-morphing origami design
Large
2 (unfolding and stretching)

Origami unfolding and

material stretching

Quasi-sequential

deployment and inflating

Highly stretchable only

$\mathrm{E}_{\mathrm{i}} 0.5 \mathrm{MPa}$

1 (only balloon-like bloating available) or

more (for pre-programming)

Selective disposition

of multiple materials

Low (i $30 \mathrm{kPa})$

i $300 \mathrm{gf}$
Poor (due to materials' non-linearity and Mullins
effect)
Slow
No

Layer mold stacking requiring sacrificial molds or high-tech multi-material 3D printing

Table 1: Comparision of the dual-origami design and dual-morphing origami design.

strain-limiting layer is almost fully unfolded, the fluidic network unfolds solely to drive the whole body to bend, accessing the regime outside of origami kinematics by utilizing flexibility of the soft material.The motion is pre-programmable through adjusting design parameters of the origami strain-limiting layer. The finite element analysis (FEA) and experimental results provide relationships between the quasi-sequential deployment and bending motion and the geometries, to select an appropriate parameter value for a given desired motion. Dual-origami soft actuators can be directly printed by a low-cost fused deposition modeling (FDM) 3D printer using flexible filaments such as thermoplastic polyurethane (TPUs) or thermoplastic elastomers (TPEs), with an introduction of a heat treatment post-processing for soft fluidic robots to leverage high pressure holds $(>200 \mathrm{kPa})$. The proposed design can generate high force $(>3 \mathrm{~kg})$, the same way other low-stretchable soft fluidic robots that can hold high fluidic pressure (e.g., pouch motors, HASEL actuators). (Acome et al., 2018; Niiyama et al., 2015) Using dual-origami soft bending actuators as deployable fingers and integrating them with a commercial suction cup, we built a versatile soft gripper that allows the selective use of two gripping mechanisms, without spatial interference between the suction cup and the fingers. Our new design method for soft robots achieved an effective spatial extension from a limited form factor, and embodies not only portability but also cooperative abilities with other mechanical elements through growth and retract. 


\section{Results}

\section{Design of Dual-origami Soft Bending Actuators}

A common six-module dual-origami soft fluidic bending actuator that is initially folded and deploys through dual-origami unfolding is shown in Figure 2A and Figure 3. Two integral components of the dual-origami design are the origami fluidic network and the origami strain-limiting layer based on conventional deployable origami architectures. We selected Miura-ori polyhedron and zigzag folded Miura-ori as the parent origami frame of two components, respectively. (Tachi and Miura, 2012) These origami architectures have large potential deployment ratios springing from their asymmetric geometries where the facets are serially connected via crease lines at each end, as well as additional advantages of flat-foldability and rigid-foldability. A singleMiura-ori polyhedron module is comprised of four parallelogram facets connected in a closed form, and when folded, each layer contains two parallelogram facets and a diamond shaped crease line loop (Figure 2B). A single-module zigzag folded Miura-ori is identical to half of the Miura-ori polyhedron, which is comprised of two parallelograms not in a closed form (Figure 2B). For soft robotic application, we modified/determined their geometry as follows: (1) For both origami architectures, we merged two parallelograms on the same layer by removing the shared crease line because the folding of the removed crease line can be replaced by the bending of the merged flexible facet. (2) The Miura-ori polyhedron's diamond shaped crease-line-loops at the top and bottom were enclosed by plates of the same shape for fluidic actuation. (3) We optionally made a V-cut at the crease lines of the zigzag folded Miura-ori to tune the folding stiffness. As shown in Figure $2 \mathrm{~A}$ and $\mathrm{B}$, the proposed dual-origami design is in the initial folded form where the zigzag folded Miura-ori strain-limiting layers are interposed between the facets of the Miura-ori polyhedron fluidic network.

Both origami components are built to be C-channel-shaped geometry in which their facets are serially connected in the direction of stacking via C-channel shaped crease lines. When bending moments are applied at the C-channel shaped crease lines, bending intensively occurs as if origami unfolding occurs. Because the applied fluidic pressure at the Miura-ori polyhedron fluidic network produces bending moments at entire crease lines directly (fluidic network) or indirectly (strain-limiting layer), dual-origami components unfold simultaneously. We defined a single module dual-origami unit as the structure consisting of a flat fluidic network with two parallel facets and a strain-limiting layer of a single facet connected to it. A multi-module dual-origami can be readily designed by the stacking module units and connecting them via C-channel-shaped crease lines.

The quasi-sequential deployment and bending behavior of a representative six-module dual-origami soft fluidic bending actuator was experimentally measured and plotted in Figure 2C (red line). This peculiar motion can be distinguished into two modes that quasi-sequentially appear in accordance with the applied fluid pressure level (Figure 2D). The first mode, a deployment-dominant mode, appears at relatively low fluid pressure $(P<40 \mathrm{kPa})$, and both the origami fluidic network and the origami strain-limiting layer unfold simultaneously. Therefore, the deployment ratio $\left(\lambda=\frac{\left(L-L_{0}\right)}{L_{0}}\right.$, where $L$ is the effective layer length and $L_{0}$ is its initial value), in response to applied fluidic pressure, rapidly increases compared to the bending angle $(\phi)$ (Figure 2E, red area). As the applied fluid pressure increases, the second mode, a bending-dominant mode appears, and the unfolding speed of origami fluidic network overwhelms the unfolding speed of the origami strain-limiting layer. This is because the strain-limiting layer is nearly completely unfolded yet the fluidic network is not, and thus $\phi$ surges while $\lambda$ slowly increases (Figure 2E, gray area). When the applied pressure is decreased, the deployed soft body retracts due to its own elasticity. We also built a conventional soft bending actuator (widely known as PneuNet design), (Ilievski et al., 2011; Mosadegh et al., 2014; Tang et al., 2020) and plotted its behavior for comparison (Figure 2B, black line). Although the initial length $L_{0}$ of the dualorigami design $(29.5 \mathrm{~mm})$ was much lower than the $L_{0}$ of the conventional design $(107 \mathrm{~mm})$, the dual-origami actuator was unfolded similarly in scale to the conventional actuator, and the bending trajectories overlapped near $16<\phi<80^{\circ}$ (the conventional design is still advantageous for large bending because of the trade-off relationship between deployment and bending, which is further discussed in section 2.2). The aspect ratio of the dual-origami bending actuators was initially 0.657 and increased up to 3 when unfolded, which is similar to the conventional soft bending actuator's aspect ratio of 2.89 (dimensions of both actuators are shown in 
Figure 4).
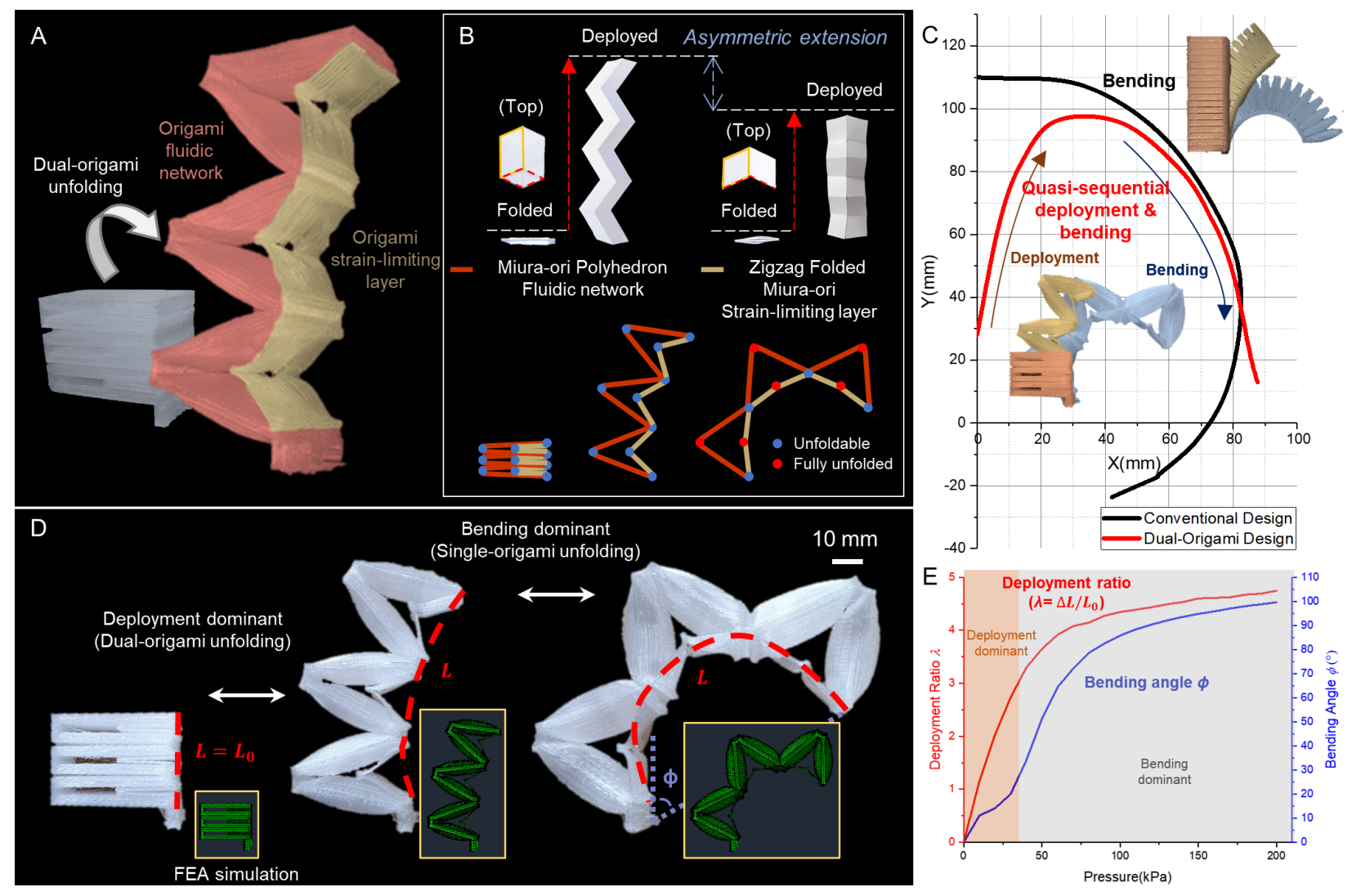

Figure 2: Design and principle of the dual-origami soft fluidic bending actuator. A) Unfolding of sixmodule dual-origami soft fluidic bending actuator. Note that the colors (red and yellow) are virtually overlaid to distinguish the components, and are actually monochromatic (white). B) Parent paper origami structures and their schematic drawings of behavior in a dual-origami soft fluidic bending actuator. C) End-tip trajectories of dual-origami soft fluidic bending actuator (red) and conventional soft pneumatic bending actuator (black). D) Quasi-sequential deployment and bending motion of six-module dual-origami soft fluidic bending actuator. Insets represent corresponding simulation result. E) Deployment ratio ( $\lambda$, red) and bending angle $(\phi$, blue) in response to applied pressure.

Rich media available at https://youtu.be/KEtq1ovht6c

\section{Pre-programing of Quasi-sequential Deployment and Bending Motion}

The bending angle of the two-module dual-origami soft fluidic bending actuator is geometrically derived as $\phi \approx\left(\theta_{\text {free }}-\theta_{\text {ori }}\right)$ and the change in effective layer length can be written as $\Delta L \approx 2 \theta_{\text {ori }} l_{\text {network }}$ (See Experimental Section/Methods for details on Simplified kinematic model. Variables are defined in Figure $5 \mathrm{~A} ; \theta_{\text {ori }}$ and $\theta_{\text {free }}$ are the angles between the two-module dual-origami soft fluidic bending actuators with an attached and unattached origami strain-limiting layer, respectively. $l_{\text {layer }}$ and $l_{\text {network }}$ are side lengths of the origami layer and the fluidic network, respectively). The ratio of the bending angle to the deployment ratio can be derived as $\frac{\phi}{\lambda}=\frac{\phi}{\Delta L / L_{0}}=\frac{L_{0}}{2 l_{\text {network }}}\left(\frac{\theta_{\text {free }}}{\theta_{\text {ori }}}-1\right)=C A_{0}$, where $A_{0}=\frac{L_{0}}{l_{\text {network }}}$ is an initial 


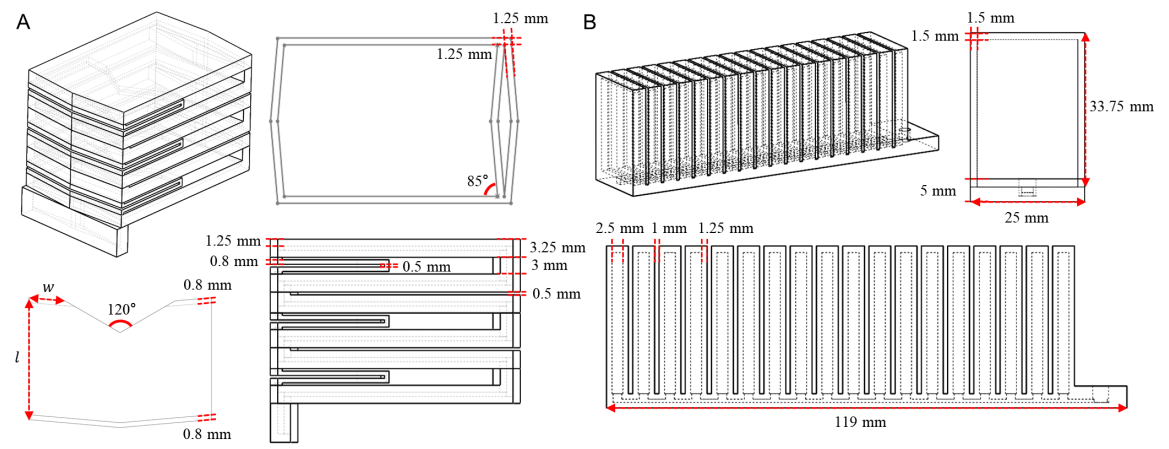

Figure 3: Dimensions of A) dual-origami soft fluidic bending actuator and B) conventional soft bending actuator.

aspect ratio and $C=\frac{1}{2}\left(\frac{\theta_{\text {free }}}{\theta_{\text {ori }}}-1\right)$. We named $C$ as the bending-to-deployment ratio factor because it represents the dominance of bending to deployment during the quasi-sequential deployment and bending motion. A high value of $C$ means that the dominance of bending is large; when the robot only bends without deployment, $C \rightarrow \infty$ (e.g., when the inextensible strain-limiting layer is attached, like conventional soft bending robots), and when the robot only deploys without bending, $C=0$ (e.g., origami fluidic network without a strain-limiting layer). For common dual-origami soft fluidic bending actuators, it was observed that $C$ rapidly increase at the transition pressure at which the dominance shift occurs (Figure 5B).

In order to better understand the quasi-sequential deployment and bending motion, we investigated the relationship between the geometric parameters of the origami strain-limiting layer and $\theta_{\text {ori }}$ or $\theta_{\text {free }}$ by performing experiments and FEA simulations (Figure 5B-E). A crease line width of one side $(w)$ and a ratio between $l_{\text {layer }}$ and $l_{\text {network }}(\Lambda)$ were considered as the important geometric parameters because it was expected that the rotational stiffness of the crease line would increase with increasing $w$ and the maximum value of $\theta_{\text {ori }}$ would be geometrically determined by $\Lambda$ (Figure $5 \mathrm{~A}$ ). In both the experiment and simulation, the response of $\theta_{\text {free }}$ to applied fluid pressure was constant even though geometric parameters of the origami layer at the nearby modules were changed (Figure $5 \mathrm{C}$ ). On the other hand, the response of $\theta_{\text {ori }}$ to applied fluid pressure was affected by $w$ and $\Lambda$. It was noteworthy that the change in $w$ gradually shifts the response while $\Lambda$ changes it significantly (Figure 5D and E). For example, the increase in $w$ from 3 to $12 \mathrm{~mm}$ decreased $\theta_{\text {ori }}$ from 58.16 to 49.56 at $100 \mathrm{kPa}$ (Figure 5D, for $\Lambda=0.476$ ), and the increase in $\Lambda$ from 0.238 to 0.476 increased $\theta_{\text {ori }}$ from 25.47 to 54.43 at $100 \mathrm{kPa}$ (Figure $5 \mathrm{E}$, for $w=6 \mathrm{~mm}$ ). The result also indicates that the amount of deployment and bending should be selectively pre-programmed with consideration of their tradeoff relationship; $\phi$ decreases as $\theta_{\text {ori }}$ increases but $\lambda$ is proportional to $\theta_{\text {or } i}$. Accordingly, $C$ was 0.158 at $20 \mathrm{kPa}$ for the most deployable case, while $C$ was 0.825 at $20 \mathrm{kPa}$ for the most bendable case, which is about 5.22 times difference (Figure 5B).

To confirm the pre-programmability of the quasi-sequential deployment and bending motion, six-module dual-origami soft fluidic bending actuators with different geometric parameters were built (Figure 5F-M). As shown in Figure 5F, J, Figure 6 and 7, we could design clear differences in motion determined by $w$ and $\Lambda$; their end-tip trajectories were plotted in Figure $5 \mathrm{G}$ and $\mathrm{K}$, respectively. $\lambda$ decreased from 4.78 to 4.32 and $\phi$ increased from 90.9 to 106.9 as $w$ increased from $3 \mathrm{~mm}$ to $12 \mathrm{~mm}$, whereas increase of $\Lambda$ from 0.238 to 0.476 greatly increased $\lambda$ from 2.35 to 4.75 and decreased $\phi$ from 144.75 to 99.66 (at $P=200 \mathrm{kPa}$, Figure $5 \mathrm{H}, \mathrm{I}, \mathrm{L}$ and $\mathrm{M}$ ). The result confirms that the quasi-sequential deployment and bending motion is more sensitive to $\Lambda$ than $w$. Therefore, it would be recommended to determine $\Lambda$ for coarse adjusting in precedence to $w$ for fine adjusting. Additionally, thickness of the strain-limiting layer $(t)$ can be considered for pre-programming of the motion. However, it should be noted that a small change in $t$ would change motion dramatically because the bending stiffness of the layer is proportional to $t^{3}$, and therefore it is not recommended to tune $t$ for motion pre-programming. (Figure 8shows simulation result that small change 
of $t=0.6 \mathrm{~mm}$ to $0.8 \mathrm{~mm}$ increases $C$ significantly from 0.115 to 0.296 at $18 \mathrm{kPa}$ ).

Rich media available at https://youtu.be/KaL5q5oI27U

Rich media available at https://youtu.be/J_f9CUrlAvM

\section{Development of Multi-Purpose Soft Gripper}

Soft grippers with soft fluidic bending actuators acting as fingers have emerged as a promising technology for safe and delicate pick-and-place including foods, pharmaceuticals, and logistics applications. With conventional soft grippers, however, the object size that can be gripped is limited by the initial configuration (Figure 9A). To widen the gripper opening, prestressed or bidirectional soft fluidic actuators were developed, (Wang et al., 2017) but the problem where the grippers should only approach the object vertically to avoid collisions still remains, which may reduce the work efficiency and increase the burden of the manipulator control and operation.

We constructed a dual-origami two-finger gripping unit where each finger at both sides is a six-module dualorigami fluidic soft fluidic bending actuator. The initial configuration was designed to be parallel to the objects to achieve a wide gripper opening (Figure 9B). The total width of the two-finger unit was relatively small compared to conventional soft robotic finger, but the finger unit could grip larger objects, even objects larger than its initial size. In addition, because the compactness greatly reduced the risk of collision, the finger unit could approach to objects via various paths (vertical, horizontal, and diagonal from bottom to top, Figure 9C).

The motion and closed configuration were pre-programmed via FEA simulation and prototyping for fast and versatile grip. When all strain-limiting layers were built with the same parameters that prefer deployment over bending (Design II of Figure 9D), the closed configuration was achieved at $100 \mathrm{kPa}$ with a $93 \mathrm{~mm}$ gap. On the other hand, when the modules at the bottom were designed to be bent greatly (Design I of Figure $9 \mathrm{D})$, the gripping unit formed a narrow-closed configuration with a $65 \mathrm{~mm}$ gap at $40 \mathrm{kPa}$. Therefore, Design I can be considered as the suitable unit that allows for fast grip with sufficient contact area during power grip (grip that wraps around an object).

The gripping force of Design I gripping unit was measured (experimental setup is shown in Figure 10 and explained in the Experimental Section). We tested gripping three PLA cylinders of different diameters, 25 $\mathrm{mm}, 50 \mathrm{~mm}$ and $75 \mathrm{~mm}$. While the gripping unit was holding the cylinders, the cylinders were pulled at a uniform speed $(3.3 \mathrm{~mm} / \mathrm{s})$. As shown in Figure 9E, the peak force appeared during power grip, and then a relatively uniform force was measured during pinch grip, and finally the cylinder was completed pulled out. At $220 \mathrm{kPa}$, the peak force of $3.15 \mathrm{kgf}, 1.53 \mathrm{kgf}$, and $1.25 \mathrm{kgf}$ were measured for $75 \mathrm{~mm}, 50 \mathrm{~mm}$, and $25 \mathrm{~mm}$ cylinders, respectively (Figure $9 \mathrm{~F}$ ). The gripping unit is lightweight $(56.8 \mathrm{~g}$ ), and the payload-to-weight ratio exceeds 55. We also tested for repeatability of the gripping unit. After 1000 -cycles at $40 \mathrm{kPa}$, the bending angle of the gripping unit was change by $8.19 \%$ compared to the 200-cycles (Figure 11).

As an application that leverages the spatial benefit of the retractable finger gripping unit, we developed a versatile soft gripper that uses both dual-origami fingers and suction gripper (VSS40-S-X, F.TEC, Figure 12A). For conventional grippers that complementarily utilize the advantages of finger grip and suction grip (in order to grip from rough and rounded object to flat and smooth object), protruding fingers interfere with the suction gripper when contacting the objects, and this problem required additional actuators and control strategies; e.g., attaching linear actuator to suction gripper. In the case of our proposed versatile gripper, the folded fingers are at the rear of the suction cup; they inherently do not interfere with the suction gripper. When the finger units are actuated, they deploy to the front of the suction gripper and can grip the object by themselves or even collaborate with the suction gripper. We attached the versatile gripper to a conventional robotic arm (M1013, Doosan Robotics) and conducted a pick-and-place task for ten objects (Figure 12B and Figure13). The soft fingers could grip various objects ranging from soft and 
light objects (e.g., 6-g mask, soft doll, confectionery) to a 2.5-kg dumbbell (Figure 12C). The suction grip was selectively used for flat objects, and fingers could be sequentially used for collaborative grasping (soup bowl). Moreover, by attaching artificial nails at the tip, the fingers could pick up a flat coin, reorient it in the upright position, and insert it in a slot precisely (Figure14 and Experimental section for detail, tasks that each requires strength, delicacy, precision and dexterity were conducted).

Rich media available at https://youtu.be/Sej0WkrWAJg

Rich media available at https://youtu.be/fziF-cVWy5A

The pre-programmability of the motion also enables customizing the dual-origami two-finger gripping unit according to the object shape. As shown in Figure 15A, we built a two-finger gripping unit customized for box-shaped objects. Two stain-limiting layers at the bottom and middle modules were designed to be long $(\Lambda=0.531, \quad w=6 \mathrm{~mm})$ while the strain-limiting layer at the top module was designed to be short $(\Lambda=$ $0.231, w=9 \mathrm{~mm})$. Consequently, the two-finger gripping unit undergoes two-step motion of first widening the gripper opening significantly (from $68 \mathrm{~mm}$ to $215 \mathrm{~mm}$ ) and then closing it moderately (Figure 15A). In the demonstration of the soft gripper with the developed two-finger gripping units, the box-shaped objects with different sizes (width of $100 \mathrm{~mm}$ to $208 \mathrm{~mm}$, Figure 15B) were picked and placed (Figure 15C, D, and Figure 16).

Rich media available at https://youtu.be/mbY8TSlohXg

\section{D printing and Heat Treatment of Dual-origami Soft Fluidic Bending Actuator}

Soft fluidic robots are principally fabricated through molding and casting of elastomers. (Polygerinos et al., 2017; Ilievski et al., 2011; Marchese et al., 2015) However, the inevitable process of detaching cured elastomer from the molds has led to difficulties in designing complex fluidic networks because the molds are usually buried within the elastomeric structure. As a solution, using sacrificial molds, made of soluble or low melting materials such as polyvinyl alcohol (PVA) and wax, were proposed.(Koivikko and Sariola, 2019) In our previous study, ${ }^{(K i m ~ e t ~ a l ., ~ 2019) ~}$ we have fabricated zigzag folded shaped origami architectures by the layer stacking method using PVA molds. However, these fabrication processes are complicated and time-consuming, while requiring experienced hands due to low material stiffnesses of the sacrificial molds. Meanwhile, 3D printing has been recently studied as an automotive fabrication method for soft robots.(Polygerinos et al., 2017; Yap et al., 2016; Low et al., 2020; Sachyani Keneth et al., 2021; Wallin et al., 2018) In particular, FDM is a prominent and popular open-source technology with economic merits of low initial and maintenance costs. The final products can be built without supports by bridging gaps within a few centimeters, and in recent studies, researchers have directly fabricated conven-

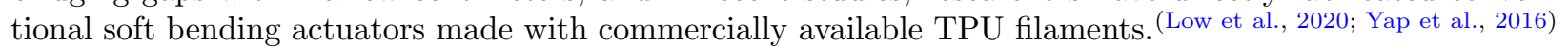
However, because FDM printers directly add molten material line by line, defects and gaps often occur at the boundary lines, leading to failure or fluid leakage at high fluid pressure.

We fabricated a dual-origami soft fluidic bending actuator using an FDM 3 printer as shown in Figure 16A. The printed actuator can be actuated immediately after gluing a urethane tubing with an adhesive, but failure or leakage occasionally happened when high pressure $(>200 \mathrm{kPa})$ was applied. For robust fabrication, we attempted a heat treatment of the fluidic soft actuator in an oven, expecting leakage prevention and enhancement of pressure holding. It was important to heat using the appropriate temperature and time; i) when either of them was insufficient, the post-processing was of little avail, and ii) when the conditions were excessive, the processed product was deformed or even melt and collapsed (Figure 17). During the process, the soft bending actuator was placed between aluminum blocks to prevent possible deformation of modules at both ends. The post-processing was successful at 172C for 2 hours (for reference, melting temperature of TPUs, $T_{m}>190 \mathrm{C}$ ), and the white or transparent TPUs turned yellowish after post-processing. As seen in micrographs in Figure 16B, it was observed that the boundaries between printed lines became blurred 
and were filled with adhered materials regardless to build direction. We verified the effectiveness by printing small test pouches and comparing maximum sealing pressure for raw and heat-treated pouches. As a result, the raw pouches could withstand $129 \mathrm{kPa}$ on average, while the heat-treated pouches could withstand 756 $\mathrm{kPa}$ on average, which is an increase of 5.58 times (Figure 17). 

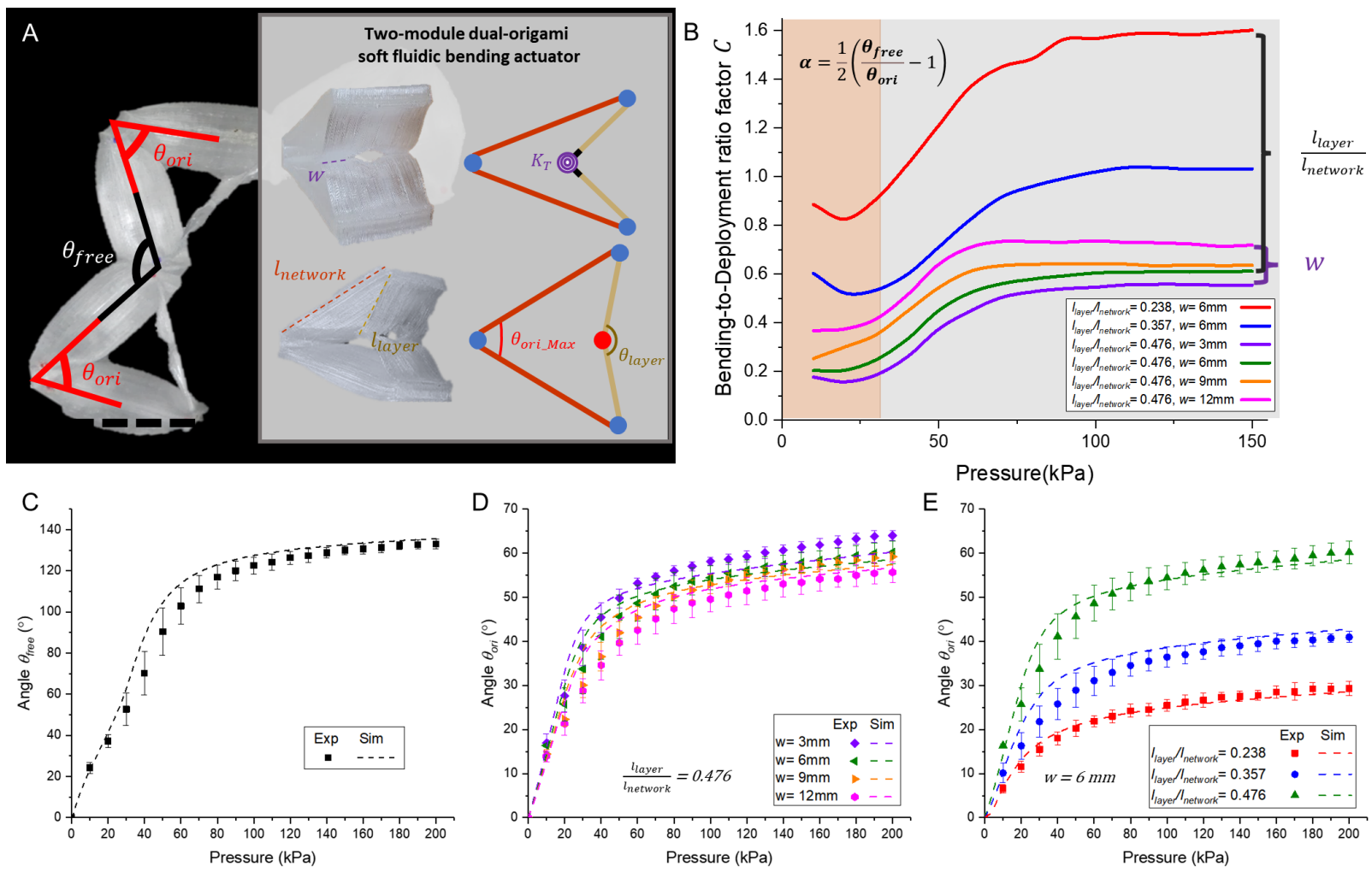

E
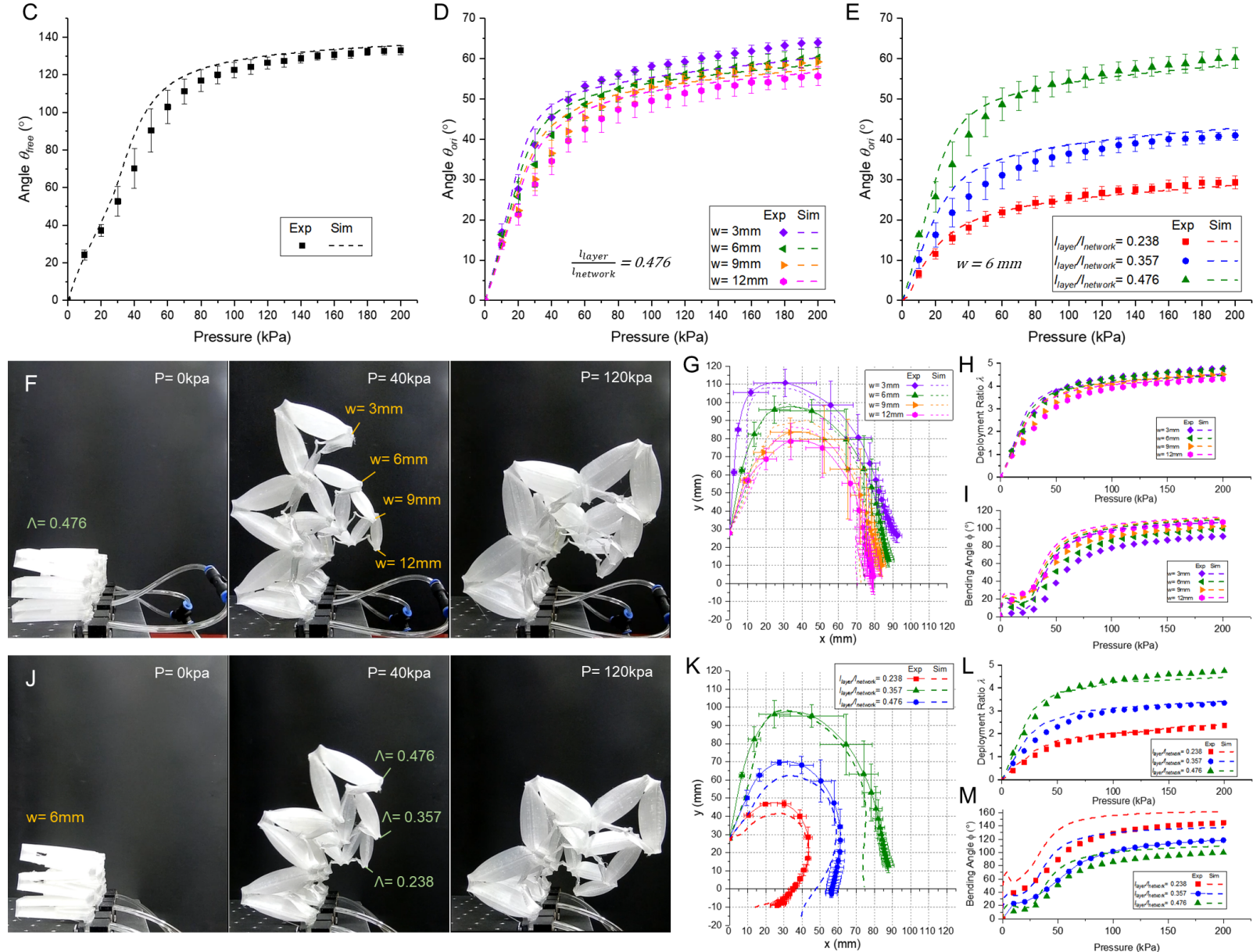

Figure 4: Parametric study of two-module dual-origami soft fluidic bending actuator and pre-programming of the quasi-sequential deployment and bending motion for six-module dual-origami soft fluidic bending actuators. A) Geometric parameters $\left(\frac{l}{L}\right.$ and $\left.w\right)$ and output angles $\left(\theta_{\text {ori }}\right.$ and $\left.\theta_{\text {free }}\right)$. B) Bending-todeployment ratio factor $(C)$ in response to applied pressure. C) Experimental and simulation results of the angle between two-modules in response to applied pressure without a strain-limiting layer, (D and E) with strain-limiting layers of different D) length ratio and E) width. F) For strain-limiting layer with different width, six-module dual-origami soft fluidic bending actuators were placed in raw and actuated simultaneously by same applied pressure. Experimental and simulation results for G) end-tip trajectories, $\mathrm{H}$ ) deployment ratio $(\lambda)$ and I) bending angle $(\phi)$ were plotted. (J, K, L, and M) Same procedure as (F-I) were performed for strain-limiting layer with different 1 length ratio $(\Lambda)$. 

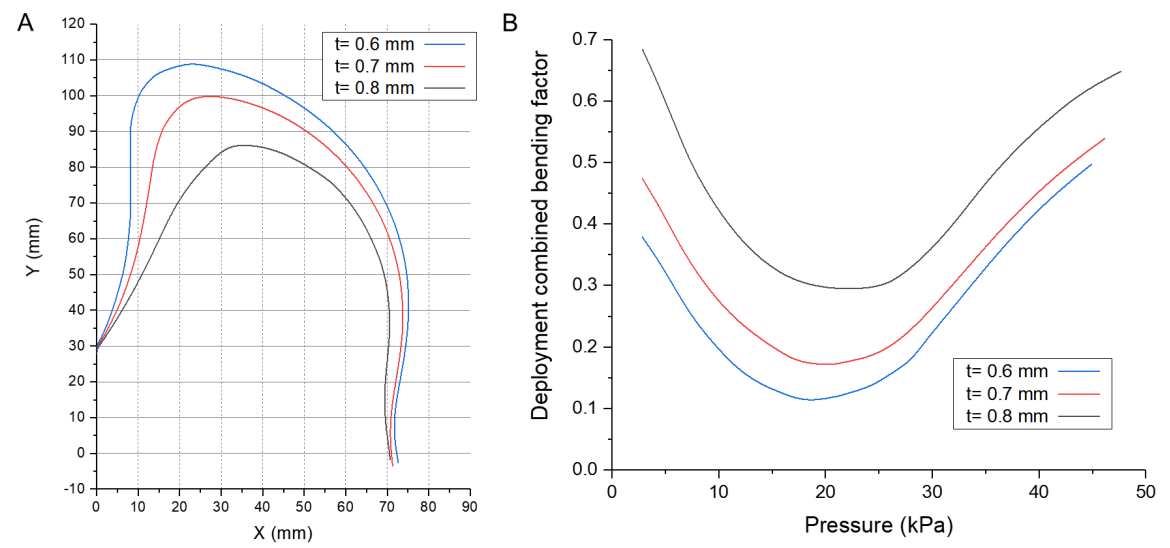

Figure 5: A parametric study of the relationship between the quasi-sequential deployment and bending motion and the thickness of the strain-limiting layer $(t)$. Data are achieved using FEA simulation. A) Endtip trajectories of the 6-module dual-origami fluidic soft actuators with variable $t$. B) Bending-to-deployment ratio factor in response to applied pressure $(<50 \mathrm{kPa}$, deployment state) with variable $t$.

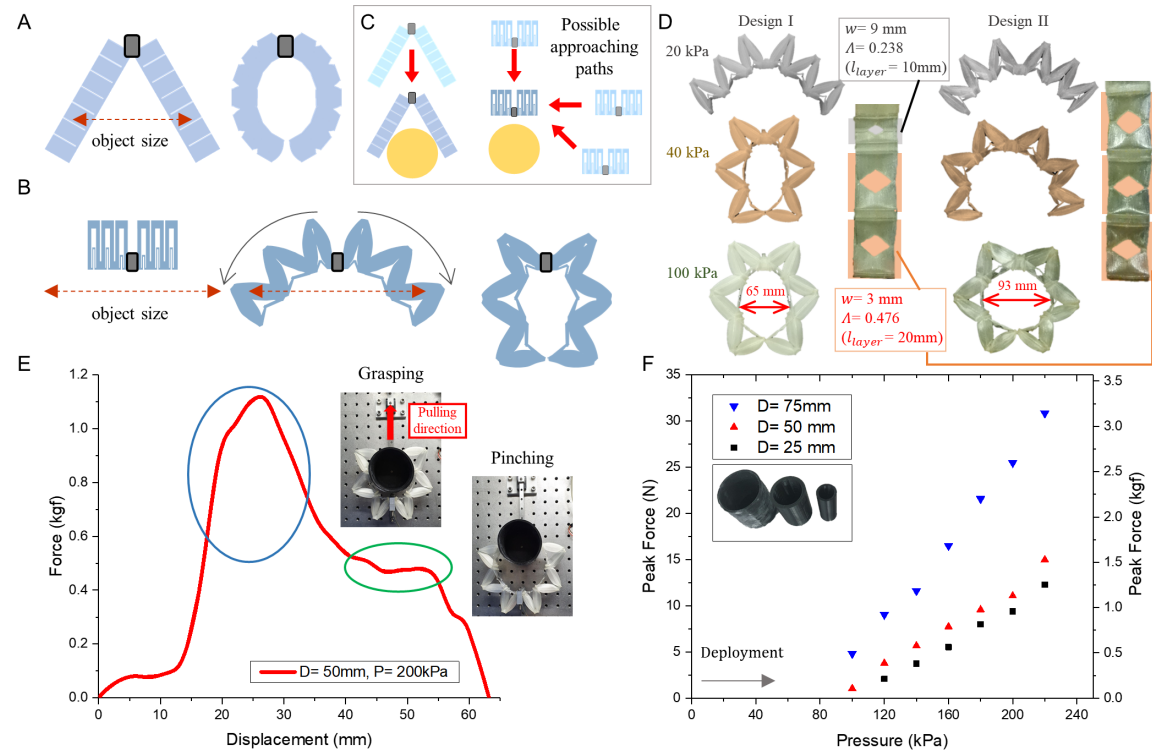

Figure 6: A dual-origami two-finger gripping unit. Gripping motion and affordable object size of A) a conventional soft gripper and B) a dual-origami two-finger gripping unit, and comparison of their C) possible approaching paths. D) Motion and configuration design of the gripping units. E) The gripping force of the Design I gripping unit $(\mathrm{P}=200 \mathrm{kPa})$ when the gripped cylinder $(\mathrm{D}=50 \mathrm{~mm})$ is pulled-out. F) Peak gripping forces of the Design I gripping unit in response to applied pressure for different cylinder diameters. 


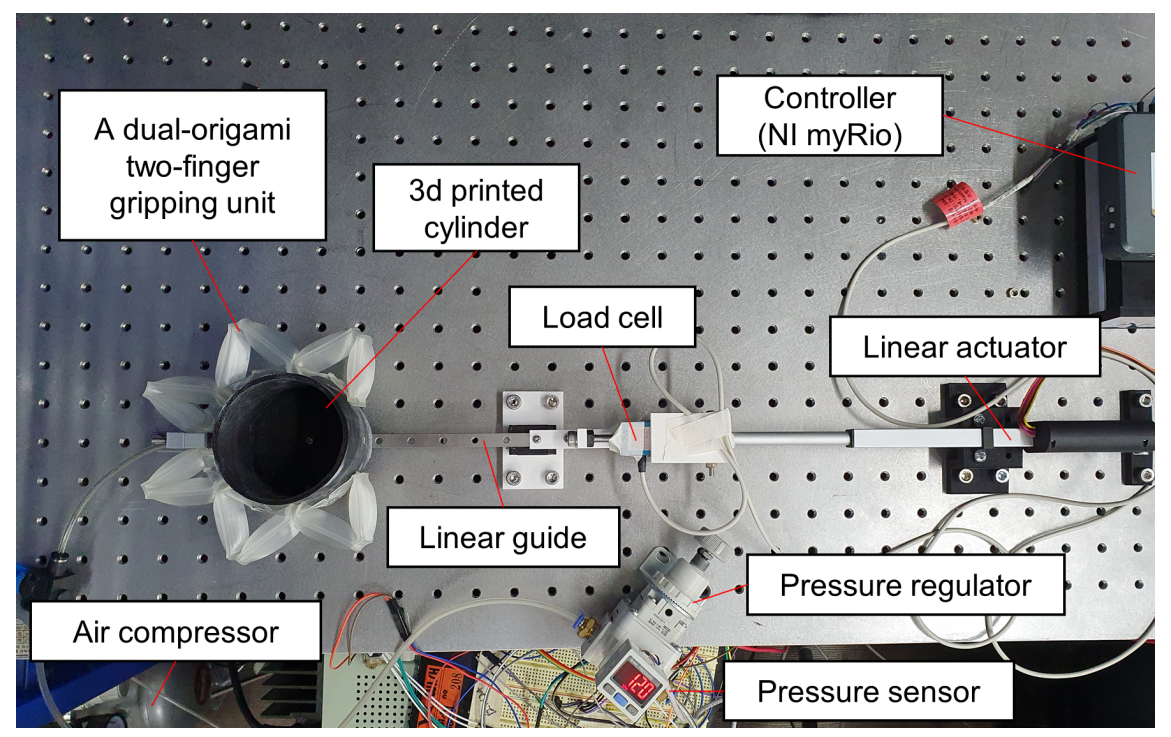

Figure 7: Experimental setup for gripping force measurement of the dual-origami two-finger gripping unit.
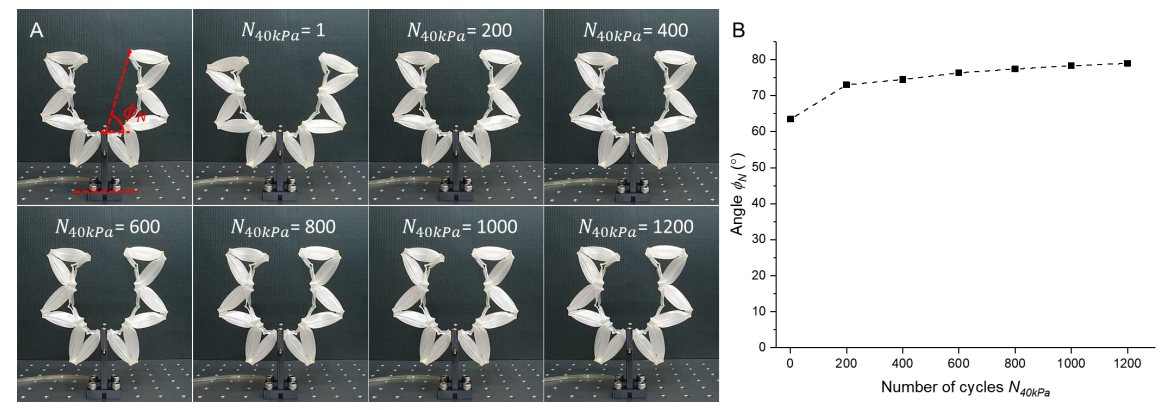

Figure 8: Repeatability test results of the dual-origami two-finger gripping unit. A) Side view of the dualorigami two-finger gripping unit at applied pressure of $40 \mathrm{kPa}$ and number of cycles $N_{40 \mathrm{kPa}}$. B) Variation of bending angle $\phi_{N}$ with the number of cycles $N_{40 \mathrm{kPa}}$. 


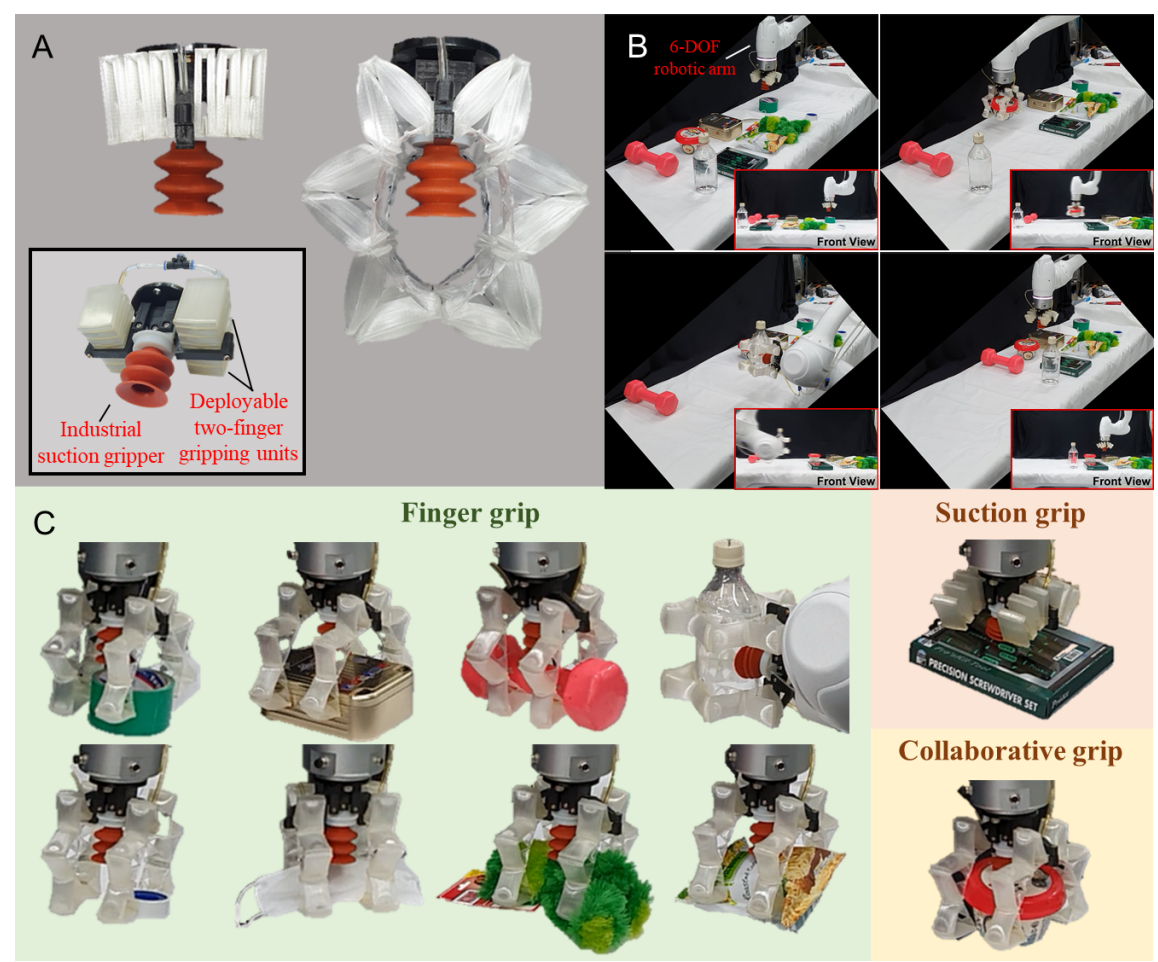

Figure 9: A versatile soft gripper. A) Overview of the developed gripper. B) Pick-and-place for various objects of irregular shapes and sizes. C) Depending on the object and environment, finger grip (green), suction grip (red), and collaborative grip (yellow) could be considered respectively.

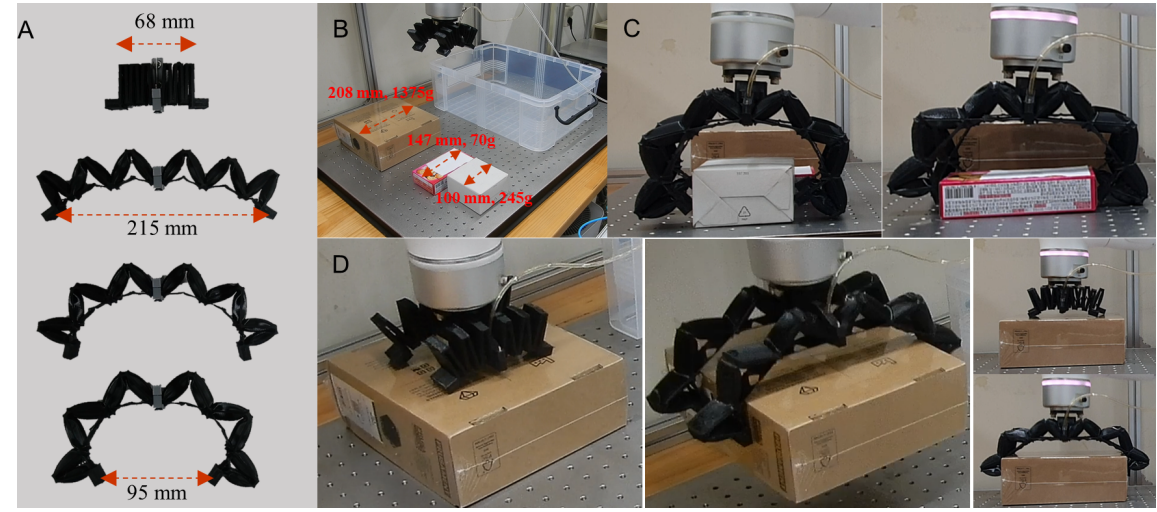

Figure 10: A soft gripper customized for box-shaped objects. A) Overview of the two-finger gripping unit for box-shaped objects. B) Box-shaped objects of different sizes. (C and D) Soft gripper picking and placing different boxes. 


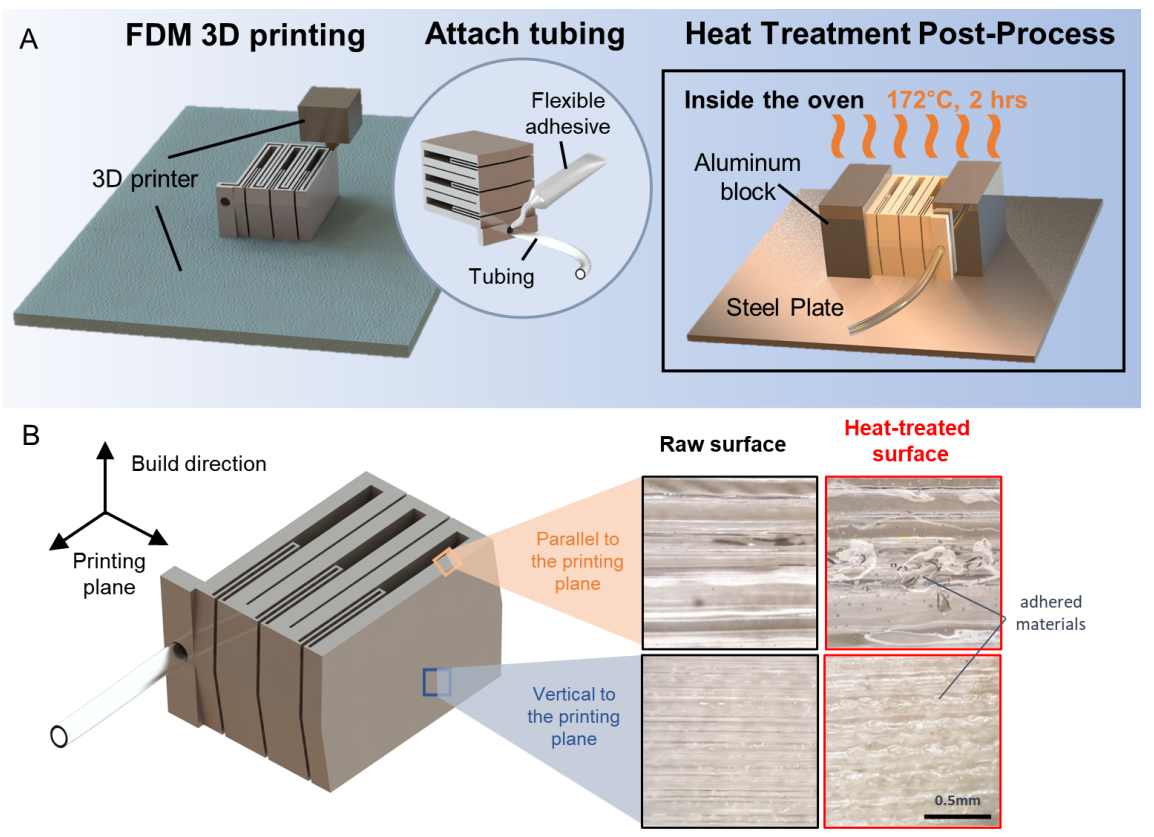

Figure 11: Fabrication and reinforcement of the dual-origami soft fluidic bending actuator. A) 3-step process of fabrication. Oven heat treatment post-process can be considered as optional if high force of the actuator is not required. B) Micrographs of raw surface and heat-treated surface.

A

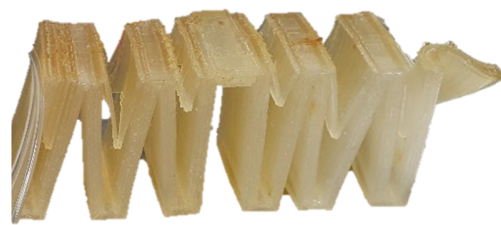

B

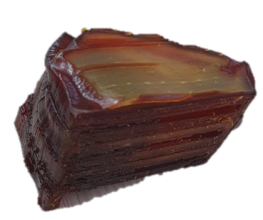

Side view

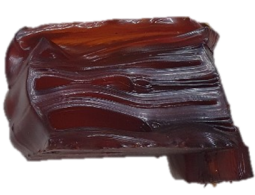

Figure 12: Failure cases of the heat treatment. A) Deformation occurred when the soft robot was heat-treated without constraining aluminum blocks. B) The soft robots melted when heat-treated at an excessively high temperature (180C 6 hours). 


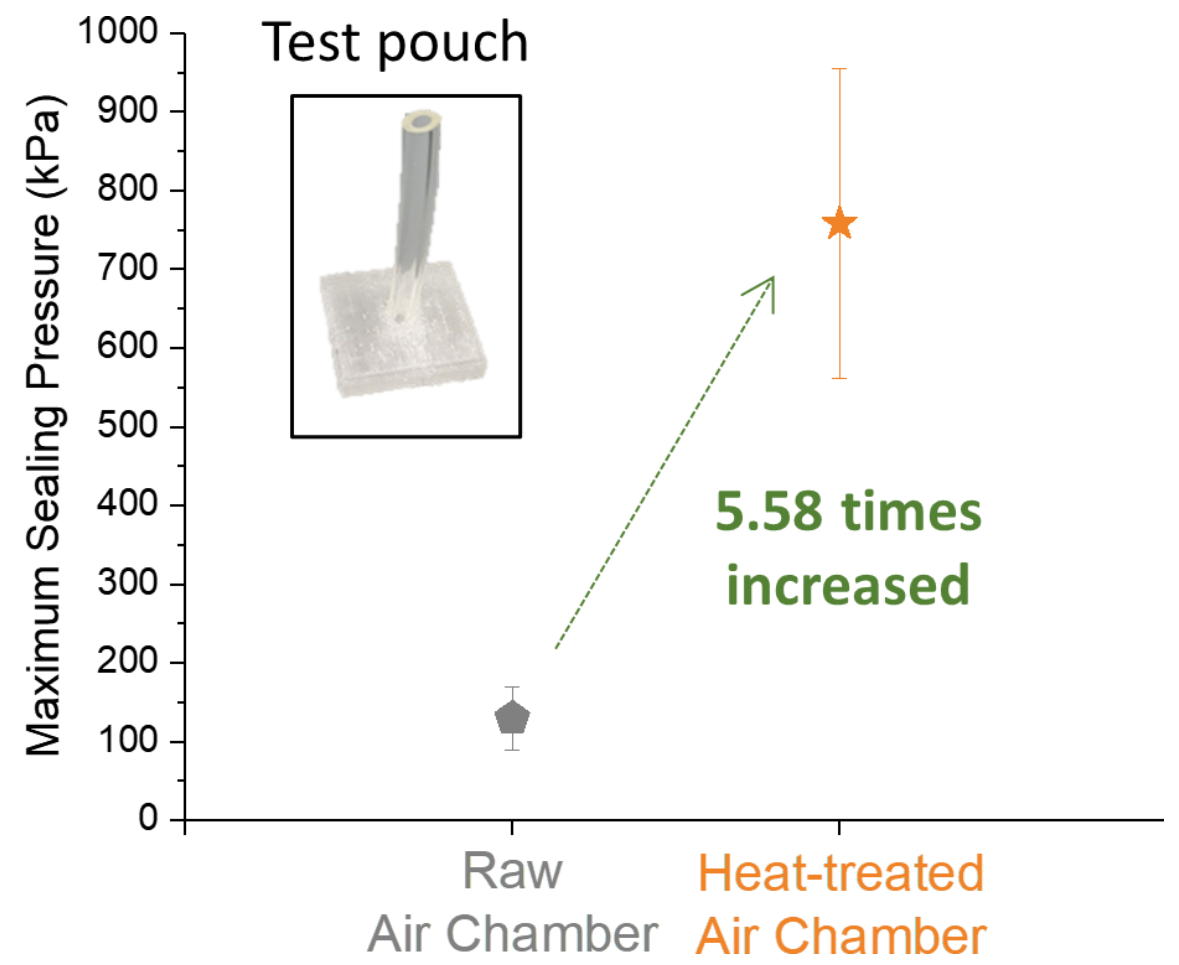

Figure 13: Maximum sealing pressure of the raw air chamber and the heat-treated air chamber. 


\section{Experimental Section/Methods}

\section{Simplified kinematic model}

The six-module dual-origami soft fluidic robot can be represented as connected linkage model, and its lengths, angles, and positions were defined as shown in Figure 20. With the assumption that the stretching of the material is negligible $\left(\mathrm{E}^{\sim} 10 \mathrm{MPa}\right.$, strain is $\sim 1 \%$ at applied pressure of $100 \mathrm{kPa}$ ), it can also be assumed that lengths $\left(L, h_{1}, h_{2}\right)$ are constant.

For positions $\overrightarrow{p_{n}}$, following relationships can be established:

$$
\begin{aligned}
& \overrightarrow{p_{0}}=\left[\begin{array}{l}
0 \\
0
\end{array}\right] \\
& p_{4 \vec{n}-3}=p_{4 \vec{n}-4}+\left[\begin{array}{c}
h_{1} \cos \beta_{2 n-1} \\
h_{1} \sin \beta_{2 n-1}
\end{array}\right] \\
& p_{4 \vec{n}-2}=p_{4 \vec{n}-3}+\left[\begin{array}{c}
L \cos \alpha_{2 n-1} \\
L \sin \alpha_{2 n-1}
\end{array}\right] \\
& p_{4 \overrightarrow{n-1}}=p_{4 \overrightarrow{n-2}}+\left[\begin{array}{c}
h_{2} \cos \beta_{2 n} \\
h_{2} \sin \beta_{2 n}
\end{array}\right] \\
& \overrightarrow{p_{4 n}}=p_{4 n-1}+\left[\begin{array}{c}
L \cos \alpha_{2 n} \\
L \sin \alpha_{2 n}
\end{array}\right]
\end{aligned}
$$

For angles $\alpha_{n}$ and $\beta_{n}$, following relationships can be established:

For $\mathrm{n}$ is odd,

$$
\begin{gathered}
\alpha_{n+1}-\alpha_{n}=\left\{\begin{array}{c}
2 \theta_{n}+\pi, \text { for } \alpha_{n}<0 \text { and } \alpha_{n+1}>0 \\
2 \theta_{n}-\pi, \text { else }
\end{array}\right. \\
\beta_{n+1}=\left\{\begin{array}{c}
-\frac{\pi}{2}+\theta_{n}+\alpha_{n}+2 \pi, \text { for } \alpha_{n}<0 \text { and } \beta_{n+1}>0 \\
-\frac{\pi}{2}+\theta_{n}+\alpha_{n}, \text { else }
\end{array}\right.
\end{gathered}
$$

For $\mathrm{n}$ is even,

$$
\begin{aligned}
& \alpha_{n+1}-\alpha_{n}=\left\{\begin{array}{c}
-2 \theta_{n}-\pi, \\
\text { for } \alpha_{n}>0 \text { and } \alpha_{n+1}<0 \\
-2 \theta_{n}+\pi, \text { else }
\end{array}\right. \\
& \beta_{n+1}=\left\{\begin{array}{c}
\frac{\pi}{2}-\theta_{n}-\alpha_{n}-2 \pi, \text { for } \alpha_{n}>0 \text { and } \beta_{n+1}<0 \\
\frac{\pi}{2}-\theta_{n}-\alpha_{n}, \text { else }
\end{array}\right.
\end{aligned}
$$


Initial conditions $\alpha_{n}$ and $\beta_{n}$ :

$$
\alpha_{1}=\pi-\theta_{0}
$$

$$
\beta_{1}=\frac{\pi}{2}(11)
$$

By solving for (6-11), angles $\alpha_{n}$ and $\beta_{n}$ can be expressed as follows:

For $n \geq 2, n \in N, m_{1}, m_{2} \in Z$,

$$
\begin{aligned}
& a_{n}=2 \sum_{k=1}^{n-1}\left\{(-1)^{k+1} \theta_{k}\right\}+\frac{1+(-1)^{n}}{2} \pi+\pi-\theta_{0}+2 \pi m_{1} \\
& \beta_{n}=-\frac{\pi}{2}+(-1)^{n}\left[2 \sum_{k=1}^{n-2}\left\{(-1)^{k+1} \cdot \theta_{k}\right\}+\theta_{n-1}+\pi-\theta_{0}\right]+2 \pi m_{2}
\end{aligned}
$$

When all modules are same, following relationship for $\theta_{n}$ is established:

$\theta_{n}=\left\{\begin{array}{c}\theta_{\text {ori }}, n \text { is odd } \\ \theta_{\text {free }}, n \text { is even }\end{array}\right.$

Finally, positions can be obtained as a function of $\theta_{n}$ by substituting (1-5) and (10-14).

For $2 \mathrm{~N}$-module structure, bending angle $\phi$ can be calculated from end-tip position $\overrightarrow{p_{4 N}}$ :

$$
\overrightarrow{p_{4 N}}=\left[\begin{array}{c}
\sum_{k=1}^{N}\left\{L\left(\cos \alpha_{2 k-1}+\cos \alpha_{2 k}\right)+h_{1} \cos \beta_{2 k-1}+h_{2} \cos \beta_{2 k}\right\} \\
\sum_{k=1}^{N}\left\{L\left(\sin \alpha_{2 k-1}+\sin \alpha_{2 k}\right)+h_{1} \sin \beta_{2 k-1}+h_{2} \sin \beta_{2 k}\right\}
\end{array}\right]
$$

In case of all modules are same and $L>>h_{1}, h_{2}, \phi$ can be calculated from (15),

$$
\begin{gathered}
\phi \approx \tan ^{-1}\left\{\frac{\sum_{k=1}^{N} \cos \alpha_{2 k-1}+\cos _{2 k}}{\sum_{k=1}^{N} \sin \alpha_{2 k-1}+\sin \alpha_{2 k}}\right\} \\
=\tan ^{-1}\left\{\frac{\sum_{k=1}^{N} \sin \left\{(2 k-1)\left(\theta_{\text {free }}-\theta_{\text {ori }}\right)\right\}}{\sum_{k=1}^{N} \cos \left\{(2 k-1)\left(\theta_{\text {free }}-\theta_{\text {ori }}\right)\right\}}\right\} \\
=N\left(\theta_{\text {free }}-\theta_{\text {ori }}\right)
\end{gathered}
$$

In Figure 20C, the model was compared with the experimental result, and it was well suited for low applied pressure.

Change in effective layer length $\Delta L$ can be approximated as a length of an arc:

$$
\Delta L \approx 2 N \theta_{\text {ori }} l_{\text {network }}
$$

A ratio of the bending angle to the deployment ratio can be derived from (16) and (17): 


$$
\begin{gathered}
\frac{\phi}{\lambda}=\frac{\phi}{\Delta L / L_{0}} \\
=\frac{L_{0}}{2 l_{\text {network }}}\left(\frac{\theta_{\text {free }}}{\theta_{\text {ori }}}-1\right) \\
=C A_{0}
\end{gathered}
$$

Where we defined bending-to deployment ratio factor $C$ as:

$$
C=\frac{1}{2}\left(\frac{\theta_{\text {free }}}{\theta_{\text {ori }}}-1\right)
$$

and $A_{0}=\frac{L_{0}}{l_{\text {network }}}$ is an initial aspect ratio.

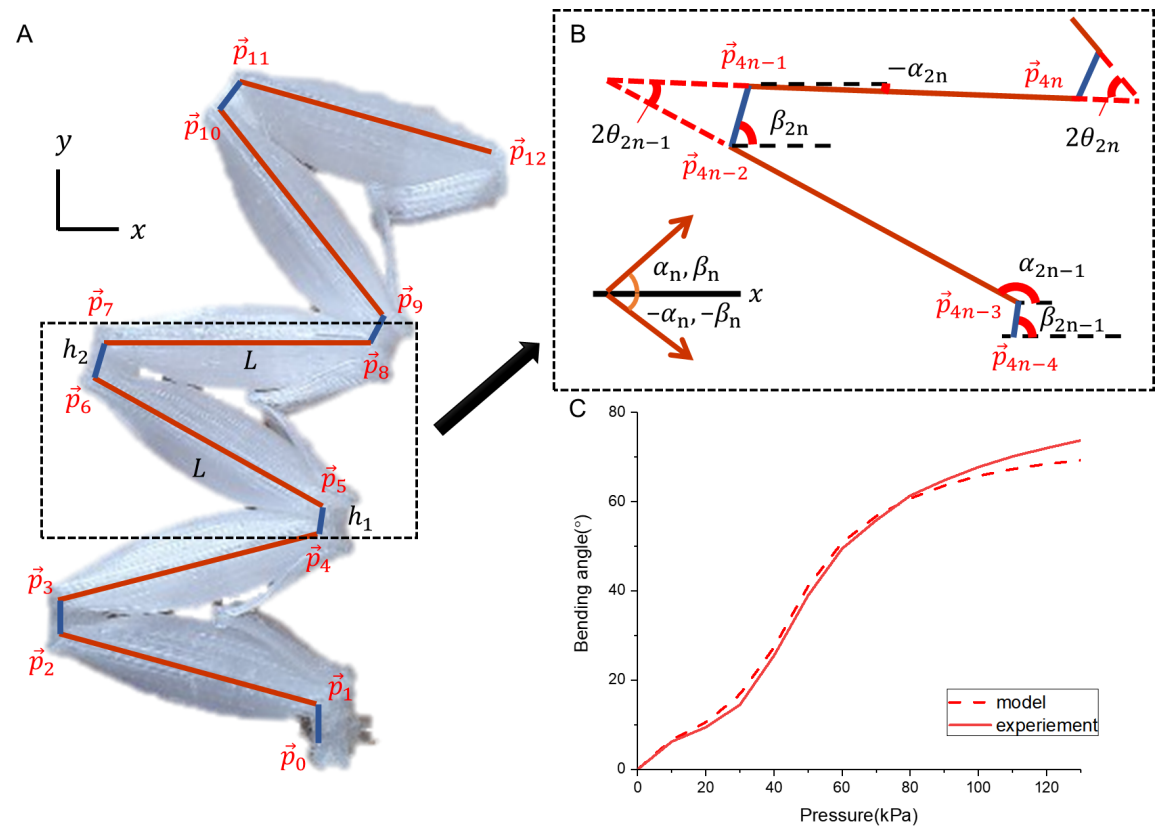

Figure 14: Linkage model for dual-origami soft fluidic actuator. A) 6-module dual-origami soft fluidic actuator's linkage model and position vectors. B) Definition of angles for calculating position. C) Comparison of model for bending angle and experimental result.

\section{D printing process}

Commercially available flexible filament eFlex (TPU, shore hardness 87A, eSUN) was used as the 3D printing material, and all experiments were conducted with robots made of this material. Other flexible materials including eTPU-95A (TPU, shore hardness 95A, eSUN), NinjaFlex (TPU, shore hardness 85A, NinjaTek), and eLastic (TPE, shore hardness 85A, eSUN) were also tested and it was confirmed that all of the tested 
materials can be used. Open-source slicing software Ultimaker Cura (Ultimaker BV) was used to prepare stereolithography (STL) files and they were imported into DIY 3D printer CORE 200 (Making Tool) using a $0.4 \mathrm{~mm}$ nozzle. For stable printing, the print speed was set as slow as $20 \mathrm{~mm} / \mathrm{s}$, and it took about 4 hours to print the six-module dual-origami and about 9 hours to print the dual-origami two-finger gripping unit. For stiffer material eTPU-95A, $40 \mathrm{~mm} / \mathrm{s}$ of print speed could be applied, and the printing time was halved. Printer performance affected the consistency of soft robots' motion because a small error in strainlimiting layer thickness (related to bending stiffness $\sim t^{3}$ ) significantly changed the dominance of deployment and bending.

\section{Characterization and Measurement:}

For the parametric study, we prepared five six-module dual-origami soft fluidic bending actuators for each geometric parameter. Red or blue dots were marked with a pen on the side edge of the modules. A protruded diamond shaped crease line loop at the bottom was surrounded and held by $3 \mathrm{~d}$ printed rigid parts, and then these rigid parts were fixed to an aluminum optical breadboard. Dual-origami soft fluidic bending actuators were installed vertically to the gravity direction to ensure that gravity does not affect the deployment. For every specimen, photographs were taken for applied pressure from 0 to $200 \mathrm{kPa}$ with $10 \mathrm{kPa}$ increments, which was precisely controlled by a pressure regulator (RVUM, PISCO). The photographs were analyzed with MATLAB (MathWorks). The center positions of the marked circles were automatically found and characterized based on the color information. The angle between adjacent modules $\left(\theta_{\text {ori }}\right.$ and $\left.\theta_{\text {free }}\right)$ was derived by calculating the angle between straight lines connecting points, and effective layer length $(L)$ was derived using cubic Hermite spline of points at strain-limiting layer side. The bending angle $(\phi)$ was derived by calculating the angle between the axis in the height direction and the line connecting the floor and the end-tip trajectory. For the gripping force measurement, a regulated constant pressure (from $100 \mathrm{kPa}$ to $220 \mathrm{kPa}$ with $20 \mathrm{kPa}$ increment for each experiments) was applied to the two-finger gripping unit holding a cylinder located at the end of the rail connected to a load cell (333FDX, KTOYO) and a linear actuator (P16-P, Actuonix Motion Devices, Inc.). To estimate the scale of friction, the rail without external gripping force was also pulled and the friction force was measured to be as low as below $50 \mathrm{gf}$.

\section{Finite Element Analysis}

Finite element analysis was conducted using FEA software ABAQUS (Dassault systems). Uniaxial tensile test for eFlex was performed following iso standard ASTM D412, and the result was imported into ABAQUS as a material property. All simulation conditions were set identical to the experimental conditions; the protruded diamond shaped crease line was set as a fixed boundary condition, and the contacts between facets were considered using the 'general contact condition'. Nodes corresponding to the points marked in the actual experiment were selected and their unique nodal displacements were collected to calculate the angle between adjacent modules, deployment ratio, and bending angle.

\section{Gripping tasks}

Figure 14 shows the soft gripper successfully conducting gripping tasks designated at the manipulation competition of Robosoft 2021. The first task requires strength to grip a heavy and large fruit (1-2 kg), and the second task requires delicacy to grip a small sized delicate fruit. Korean radish weighing $1.3 \mathrm{~kg}$ and raspberry were chosen respectively. The third and fourth tasks were to pick a coin an insert it into a coin slot, requiring precision and dexterity respectively. The Korean 100-won coin (24 mm diameter) was chosen and the coin slot was fabricated as $4 \mathrm{~mm} \mathrm{X} 27 \mathrm{~mm}$ using an acrylic plate. 


\section{Conclusion}

In this paper, we have presented a soft fluidic bending actuator that undergoes characteristic quasi-sequential deployment and bending motion, attributed by an asymmetric extension of dual-origami components; a folded fluidic chamber and a folded strain-limiting layer. The proposed design embodies spatial benefits of deployment and retraction that originate from origami architectures, as well as inherent adaptiveness of soft fluidic robots. The dual-origami soft fluidic bending actuators can be directly and automatically fabricated through accessible 3D printing technology, and additional heat treatment post-processing was introduced to reinforce the material adhesion. Furthermore, we have investigated the kinematic features of the flexible soft origami robot and established a bending-to-deployment ratio factor to quantify the dominance shifting from deployment to bending. The relationship between design parameters and the motion was also explored and the tradeoff relationship between deployment and bending was demonstrated through pre-programming of six-module dual-origami fluidic bending actuators. Moreover, we built the deployable two-finger gripping unit that can generate high holding force $(\sim 3.15 \mathrm{~kg}$, weight $56.8 \mathrm{~g})$, and applied them into a versatile soft gripper that cooperate with a commercial suction gripper. The demonstration has successfully shown that the deployable finger units can not only grow and adapt to various objects, but also can make place to the suction cup for efficient use of the entire system.

We believe that our approach provides a new design guidance of soft fluidic robots to embody the grow-andretract motion with a small initial form factor that considers space usage, differentiating from conventional design methods that primarily focus on motion generation. In addition, the proposed design principle is implemented by non-stretchable and flexible materials, allowing wide range of material choice compared to our previous work using stretchable materials. (Katzschmann et al., 2018; Heng et al., 2021; Cianchetti et al., 2014) Future work may include fabrication using light and thin materials such as thermoplastic sheets (e.g., polypropylene, polyethylene), composite fabrics, and papers, to improve scalability and functionality. Further improvement such as sensor integration, real-time control and analytical modeling would complement the complexity of the combined motion of deployment and bending. We expect that the unique property of a small form factor soft robot with grow-and-retract motion would be considered as a powerful option for applications in next-generation soft robotic systems, including portable or mobile application, medical devices, wearable robots, and integrated robotic systems.

\section{Acknowledgements}

This work was supported by the National Research Foundation of Korea (NRF) (NRF-2016R1A5A1938472) and the Technology Program (20008908) funded by the Ministry of Trade, Industry \& Energy (MOTIE, Korea).

\section{Conflict of interest}

The authors declare no conflict of interest. 


\section{References}

E Acome, SK Mitchell, TG Morrissey, MB Emmett, C Benjamin, M King, M Radakovitz, and C Keplinger. Hydraulically amplified self-healing electrostatic actuators with muscle-like performance. Science, 359: 61-65, Jan 2018.

Sang Min Baek, Sojung Yim, Soo Hwan Chae, Dae Young Lee, and Kyu Jin Cho. Ladybird beetle-inspired compliant origami. Science Robotics, 5(41):eaaz6262, 2020. ISSN 24709476. doi: 10.1126/SCIROBOTICS. AAZ6262.

Kaitlyn P. Becker, Yufeng Chen, and Robert J. Wood. Mechanically Programmable Dip Molding of High Aspect Ratio Soft Actuator Arrays. Advanced Functional Materials, 30(12):1908919, 2020. ISSN 16163028. doi: 10.1002/adfm.201908919.

E. Brown, N. Rodenberg, J. Amend, A. Mozeika, E. Steltz, M. R. Zakin, H. Lipson, and H. M. Jaeger. Universal robotic gripper based on the jamming of granular material. Proceedings of the National Academy of Sciences, 107(44):18809-18814, oct 2010. doi: 10.1073/pnas.1003250107. URL https://doi.org/10. 1073\%2Fpnas. 1003250107.

Bohan Chen, Zhuyin Shao, Zhexin Xie, Jiaqi Liu, Fei Pan, Liwen He, Li Zhang, Yanming Zhang, Xuechen Ling, Fujun Peng, Weidong Yun, and Li Wen. Soft Origami Gripper with Variable Effective Length. page 2000251, 2021. doi: 10.1002/aisy.202000251.

Matteo Cianchetti, Tommaso Ranzani, Giada Gerboni, Thrishantha Nanayakkara, Kaspar Althoefer, Prokar Dasgupta, and Arianna Menciassi. Soft Robotics Technologies to Address Shortcomings in Today's Minimally Invasive Surgery: The STIFF-FLOP Approach. Soft Robotics, 1(2):122-131, jun 2014. doi: 10.1089/soro.2014.0001. URL https://doi.org/10.1089\%2Fsoro.2014.0001.

Fionnuala Connolly, Panagiotis Polygerinos, Conor J. Walsh, and Katia Bertoldi. Mechanical programming of soft actuators by varying fiber angle. Soft Robotics, 2(1):26-32, 2015. ISSN 21695180. doi: 10.1089/ soro.2015.0001.

Julia Deiters, Wojciech Kowalczyk, and Tobias Seidl. Simultaneous optimisation of earwig hindwings for flight and folding. Biology Open, 5(5):638-644, 2016. ISSN 20466390. doi: 10.1242/bio.016527.

Jakob A. Faber, Andres F. Arrieta, and André R. Studart. Bioinspired spring origami. Science, 359(6382): 1386-1391, 2018. ISSN 10959203. doi: 10.1126/science.aap7753.

Sandhya Rani Goudu, Immihan Ceren Yasa, Xinghao Hu, Hakan Ceylan, Wenqi Hu, and Metin Sitti. Biodegradable Untethered Magnetic Hydrogel Milli-Grippers. Advanced Functional Materials, 30(50): 2004975, sep 2020. doi: 10.1002/adfm.202004975. URL https://doi.org/10.1002\%2Fadfm. 202004975.

Elliot W. Hawkes, Laura H. Blumenschein, Joseph D. Greer, and Allison M. Okamura. A soft robot that navigates its environment through growth. Science Robotics, 2(8), jul 2017. doi: 10.1126/scirobotics. aan3028. URL https://doi.org/10.1126\%2Fscirobotics. aan3028.

Wenzheng Heng, Geng Yang, Gaoyang Pang, Zhiqiu Ye, Honghao Lv, Juan Du, Guodong Zhao, and Zhibo Pang. Fluid-Driven Soft CoboSkin for Safer Human-Robot Collaboration: Fabrication and Adaptation. Advanced Intelligent Systems, 3(3):2170030, mar 2021. doi: 10.1002/aisy.202170030. URL https://doi. org/10.1002\%2Faisy. 202170030.

Filip Ilievski, Aaron D. Mazzeo, Robert F. Shepherd, Xin Chen, and George M. Whitesides. Soft Robotics for Chemists. Angewandte Chemie, 123(8):1930-1935, 2011. ISSN 1521-3757. doi: 10.1002/ange.201006464.

Hyunki In, Brian Byunghyun Kang, MinKi Sin, and Kyu-Jin Cho. Exo-Glove: A Wearable Robot for the Hand with a Soft Tendon Routing System. IEEE Robotics $\&$ Automation Magazine, 22(1):97-105, mar 2015. doi: 10.1109/mra.2014.2362863. URL https://doi.org/10.1109\%2Fmra.2014.2362863. 
Meredith Johnson, Yue Chen, Sierra Hovet, Sheng Xu, Bradford Wood, Hongliang Ren, Junichi Tokuda, and Zion Tsz Ho Tse. Fabricating biomedical origami: a state-of-the-art review, 2017. ISSN 18616429.

Erina B. Joyee and Yayue Pan. A Fully Three-Dimensional Printed Inchworm-Inspired Soft Robot with Magnetic Actuation. Soft Robotics, 6(3):333-345, jun 2019. doi: 10.1089/soro.2018.0082. URL https: //doi.org/10.1089\%2Fsoro.2018.0082.

RK Katzschmann, J DelPreto, R MacCurdy, and D Rus. Exploration of underwater life with an acoustically controlled soft robotic fish. Sci Robot, 3, Mar 2018.

Suk Jun Kim, Dae Young Lee, Gwang Pil Jung, and Kyu Jin Cho. An origami-inspired, self-locking robotic arm that can be folded flat. Science Robotics, 3(16):eaar2915, 2018. ISSN 24709476. doi: 10.1126/ scirobotics.aar2915.

Woongbae Kim, Junghwan Byun, Jae Kyeong Kim, Woo Young Choi, Kirsten Jakobsen, Joachim Jakobsen, Dae Young Lee, and Kyu Jin Cho. Bioinspired dual-morphing stretchable origami. Science Robotics, 4 (36):eaay3493, 2019. ISSN 24709476. doi: 10.1126/scirobotics.aay3493.

Anastasia Koivikko and Veikko Sariola. Fabrication of soft devices with buried fluid channels by using sacrificial 3D printed molds. In 2019 2nd IEEE International Conference on Soft Robotics (RoboSoft), pages 509-513, Seoul, 2019. doi: 10.1109/ROBOSOFT.2019.8722741.

Shoichiro Koizumi, Shunichi Kurumaya, Hiroyuki Nabae, Gen Endo, and Koichi Suzumori. Braiding thin McKibben muscles to enhance their contracting abilities. IEEE Robotics and Automation Letters, 3(4): 3240-3246, 2018. ISSN 23773766. doi: 10.1109/LRA.2018.2851025.

Dae-Young Lee, Jae-Kyeong Kim, Chang-Young Sohn, Jeong-Mu Heo, and Kyu-Jin Cho. High-load capacity origami transformable wheel. Science Robotics, 6(53):eabe0201, 2021. doi: 10.1126/scirobotics.abe0201.

Shuguang Li, Daniel M. Vogt, Daniela Rus, and Robert J. Wood. Fluid-driven origami-inspired artificial muscles. Proceedings of the National Academy of Sciences of the United States of America, 114(50): 13132-13137, 2017a. ISSN 10916490. doi: 10.1073/pnas.1713450114.

Tiefeng Li, Guorui Li, Yiming Liang, Tingyu Cheng, Jing Dai, Xuxu Yang, Bangyuan Liu, Zedong Zeng, Zhilong Huang, Yingwu Luo, Tao Xie, and Wei Yang. Fast-moving soft electronic fish. Science Advances, 3(4), apr 2017b. doi: 10.1126/sciadv.1602045. URL https://doi.org/10.1126\%2Fsciadv. 1602045.

Yangqiao Lin, Geng Yang, Yuwen Liang, Chao Zhang, Wei Wang, Dahong Qian, Huayong Yang, and Jun Zou. Controllable Stiffness Origami "Skeletons" for Lightweight and Multifunctional Artificial Muscles. Advanced Functional Materials, 30(31):2000349, 2020. ISSN 16163028. doi: 10.1002/adfm.202000349.

J. H. Low, J. Y. Goh, N. Cheng, P. M. Khin, Q. Q. Han, and C. H. Yeow. A Bidirectional 3D-printed Soft Pneumatic Actuator and Graphite-based Flex Sensor for Versatile Grasping. In 2020 IEEE International Conference on Robotics and Automation (ICRA), pages 7979-7985, Paris, 2020. doi: 10.1109/ICRA40945. 2020.9196837.

Mariangela Manti, Taimoor Hassan, Giovanni Passetti, Nicolò D'Elia, Cecilia Laschi, and Matteo Cianchetti. A Bioinspired Soft Robotic Gripper for Adaptable and Effective Grasping. Soft Robotics, 2(3):107-116, sep 2015. doi: 10.1089/soro.2015.0009. URL https://doi.org/10.1089\%2Fsoro.2015.0009.

AD Marchese, RK Katzschmann, and D Rus. A Recipe for Soft Fluidic Elastomer Robots. Soft Robot, 2: 7-25, Mar 2015.

Ramses V. Martinez, Carina R. Fish, Xin Chen, and George M. Whitesides. Elastomeric origami: Programmable paper-elastomer composites as pneumatic actuators. Advanced Functional Materials, 22(7): 1376-1384, 2012. ISSN 1616301X. doi: 10.1002/adfm.201102978.

Stefano Mintchev, Jun Shintake, and Dario Floreano. Bioinspired dual-stiffness origami. Science Robotics, 3(20):eaau0275, 2018. ISSN 24709476. doi: 10.1126/scirobotics.aau0275. 
Bobak Mosadegh, Panagiotis Polygerinos, Christoph Keplinger, Sophia Wennstedt, Robert F. Shepherd, Unmukt Gupta, Jongmin Shim, Katia Bertoldi, Conor J. Walsh, and George M. Whitesides. Pneumatic Networks for Soft Robotics that Actuate Rapidly. Advanced Functional Materials, 24(15):2163-2170, jan 2014. doi: 10.1002/adfm.201303288. URL https://doi.org/10.1002\%2Fadfm. 201303288.

Ryuma Niiyama, Xu Sun, Cynthia Sung, Byoungkwon An, Daniela Rus, and Sangbae Kim. Pouch motors: Printable soft actuators integrated with computational design. Soft Robotics, 2(2):59-70, 2015. ISSN 21695180. doi: 10.1089/soro.2014.0023.

Nathan A. Pehrson, Daniel C. Ames, Samuel P. Smith, Spencer P. Magleby, and Manan Arya. Self-deployable, self-stiffening, and retractable origami-based arrays for spacecraft. AIAA Journal, 58(7):3221-3228, 2020. ISSN 00011452. doi: 10.2514/1.J058778.

Panagiotis Polygerinos, Nikolaus Correll, Stephen A. Morin, Bobak Mosadegh, Cagdas D. Onal, Kirstin Petersen, Matteo Cianchetti, Michael T. Tolley, and Robert F. Shepherd. Soft Robotics: Review of FluidDriven Intrinsically Soft Devices; Manufacturing, Sensing, Control, and Applications in Human-Robot Interaction. Advanced Engineering Materials, 19(12):1700016, 2017. ISSN 15272648. doi: 10.1002/adem. 201700016.

Mark Runciman, Ara Darzi, and George P. Mylonas. Soft Robotics in Minimally Invasive Surgery. Soft Robotics, 6(4):423-443, aug 2019. doi: 10.1089/soro.2018.0136. URL https://doi .org/10.1089\%2Fsoro. 2018.0136.

Ela Sachyani Keneth, Alexander Kamyshny, Massimo Totaro, Lucia Beccai, and Shlomo Magdassi. 3D Printing Materials for Soft Robotics. Advanced Materials, 33(19):1-17, 2021. ISSN 15214095. doi: 10. 1002/adma.202003387.

Kazuya Saito, Shuhei Nomura, Shuhei Yamamoto, Ryuma Niyama, and Yoji Okabe. Investigation of hindwing folding in ladybird beetles by artificial elytron transplantation and microcomputed tomography. Proceedings of the National Academy of Sciences of the United States of America, 114(22):5624-5628, 2017. ISSN 10916490. doi: 10.1073/pnas.1620612114.

Mark Schenk, Andrew D. Viquerat, Keith A. Seffen, and Simon D. Guest. Review of inflatable booms for deployable space structures: Packing and rigidization, 2014. ISSN 15336794.

D Shah, B Yang, S Kriegman, M Levin, J Bongard, and R Kramer-Bottiglio. Shape Changing Robots: Bioinspiration, Simulation, and Physical Realization. Adv Mater, 33:e2002882, May 2021.

Hiroyuki Suzuki and Robert J. Wood. Origami-inspired miniature manipulator for teleoperated microsurgery. Nature Machine Intelligence, 2(8):437-446, 2020. ISSN 25225839. doi: 10.1038/s42256-020-0203-4. URL http://dx.doi.org/10.1038/s42256-020-0203-4.

Tomohiro Tachi and Koryo Miura. Rigid-foldable cylinders and cells. Journal of the International Association for Shell and Spatial Structures, 53(174):217-226, 2012. ISSN 1028365X.

Yichao Tang, Yinding Chi, Jiefeng Sun, Tzu Hao Huang, Omid H. Maghsoudi, Andrew Spence, Jianguo Zhao, Hao Su, and Jie Yin. Leveraging elastic instabilities for amplified performance: Spine-inspired high-speed and high-force soft robots. Science Advances, 6(19):eaaz6912, may 2020. ISSN 23752548. doi: 10.1126/sciadv.aaz6912.

NS Usevitch, ZM Hammond, M Schwager, AM Okamura, EW Hawkes, and S Follmer. An untethered isoperimetric soft robot. Sci Robot, 5, Mar 2020.

Vishesh Vikas, Eliad Cohen, Rob Grassi, Canberk Sozer, and Barry Trimmer. Design and Locomotion Control of a Soft Robot Using Friction Manipulation and Motor-Tendon Actuation. IEEE Transactions on Robotics, 32(4):949-959, aug 2016. doi: 10.1109/tro.2016.2588888. URL https://doi.org/10.1109\% 2Ftro.2016.2588888. 
T. J. Wallin, J. Pikul, and R. F. Shepherd. 3D printing of soft robotic systems, 2018. ISSN 20588437.

Zhongkui Wang, Yuuki Torigoe, and Shinichi Hirai. A Prestressed Soft Gripper: Design, Modeling, Fabrication, and Tests for Food Handling. IEEE Robotics and Automation Letters, 2(4):1909-1916, 2017. ISSN 23773766. doi: 10.1109/LRA.2017.2714141.

Zhiguang Xing, Junming Zhang, David McCoul, Yanzhen Cui, Lining Sun, and Jianwen Zhao. A SuperLightweight and Soft Manipulator Driven by Dielectric Elastomers. Soft Robotics, 7(4):512-520, aug 2020. doi: 10.1089/soro.2018.0134. URL https://doi.org/10.1089\%2Fsoro.2018.0134.

Dian Yang, Mohit S. Verma, Elton Lossner, Duncan Stothers, and George M. Whitesides. Negative-Pressure Soft Linear Actuator with a Mechanical Advantage. Advanced Materials Technologies, 2(1):1600164, 2017. ISSN 2365709X. doi: 10.1002/admt.201600164.

Hong Kai Yap, Hui Yong Ng, and Chen Hua Yeow. High-Force Soft Printable Pneumatics for Soft Robotic Applications. Soft Robotics, 3(3):144-158, 2016. ISSN 21695180. doi: 10.1089/soro.2016.0030. 